\title{
Collaborative Multimedia Systems: Synthesis of Media Objects*
}

\author{
Kasım S. Candan, V.S. Subrahmanian \\ Department of Computer Science \\ University of Maryland \\ College Park, Maryland 20742. \\ $\{$ candan, vs $\}$ @cs.umd.edu
}

\author{
P. Venkat Rangan \\ Department of Computer Science \\ University of California at San Diego \\ La Jolla, CA 92093. \\ venkat@cs.ucsd.edu.
}

\begin{abstract}
When a group $\left\{I_{1}, \ldots, I_{n}\right\}$ of individuals wishes to collaboratively construct a complex multimedia document, the first requirement is that they be able to manipulate media-objects created by one another. For instance, if individual $I_{j}$ wishes to access some media objects present at participant $I_{k}$ 's site, he must be able to; (1) retrieve this object from across the network, (2) ensure that the object is in a form that is compatible with the viewing/editing resources he has available at his node, and (3) ensure that the object has the desired quality (such as image size and resolution). Furthermore, he must be able to achieve these goals at the lowest possible cost. In this paper, we develop a theory of media objects, and present optimal algorithms for collaborative object sharing/synthesis of the sort envisaged above. We then extend the algorithms to incorporate quality constraints (such as image size) as well as distribution across multiple nodes. The theoretical model is validated by an experimental implementation that supports the theoretical results.
\end{abstract}

\section{Introduction}

Collaborative multimedia systems consist of collaborators constructing and manipulating various kinds of media objects, such as video-clips, pictures, text files, or perhaps some complex entity constructed out of these simpler entities. By and large, these collaborators are located at various points across the network. When considering collaborative media systems, a vast number of different factors come into play:

- First and foremost, each collaborator must have the ability to access and manipulate the objects that he needs to access in order to fulfil his role in the collaboration. This simple step involves far more than just retrieving the object from a remote node - it involves accessing the object and routing it through a set (possibly empty) of intermediate nodes in such a way that the object, when it arrives, has the desired properties (e.g. be in a format compatible with the resources at the destination node, having a minimal desired quality, etc.). This may require not only actions at the remote node and the destination nodes, but the intermediate nodes as well.

- Second, when multiple collaborators are working together, in a collaborative group-session, then the sharing of these objects must be done in real-time, and editing changes made by one collaborator must be reflected, in a synchronized fashion, and in real-time, on the screens/output

\footnotetext{
*This research was supported by the Army Research Office under grant DAAL-03-92-G-0225, by the Air Force Office of Scientific Research under grant F49620-93-1-0065, by ARPA/Rome Labs contract Nr. F30602-93-C-0241 (Order Nr. A716), and by an NSF Young Investigator award IRI-93-57756.
} 
devices of others. Most current systems that implement such schemes (e.g. the Sun ShowMe repertoire of products) require that all nodes in the collaborative enterprise have certain common products available on them, (viz. the Sun Show Me system).

The first step listed above is a criticial pre-cursor to the second step. There is, after all, little point in synchronizing the transmission of objects if one of the collaborators cannot view the object in its current form (e.g. he may lack a given video player required to present a video object). In this paper, we focus in on one aspect of collaborative multimedia systems, viz. the first point listed above. The primary contributions we make are the following:

1. We provide a formal declarative definition of a media-object and use this as the basis of a formal definition of a collaborative media system (COMS) that involves multiple collaborators located at dispersed sites on a network such as the Internet. This definition includes not just a declarative specification of the location of different media-objects, but also of the capabilities available at these locations.

2. We provide a formal declarative definition of an object synthesis sequence that may be used by a given collaborator to obtain an object from another node in a form that he can use at his local node. This form may include not just a format specification, but also quality specifications. This is particularly important in the case of multimedia data where lossy compression techniques are frequently used, as well as where conversions from one format to another may degrade quality.

3. We provide a declarative framework for associating costs with such object synthesis sequences this automatically induces a definition of an optimal synthesis sequence.

4. The declarative specifications listed above provide a formal specification against which the correctness and optimality of algorithms can be measured.

5. Subsequently, we develop two algorithms to construct synthesis sequences - the OSA algorithm which computes a syntheseis sequence, but not necessarily an optimal one, and the OptOSA algorithm that is guaranteed to compute an optimal synthesis sequence. We formally prove that these algorithms are sound, complete and optimal (in the case of OptOSA).

6. Both the OSA and the OptOSA algorithms assume that all object synthesis is done within a single node. We then define the notion of a distributed synthesis sequence and develop two algorithms, the DOSA and DOptOSA algorithms that extend the OSA and the OptOSA algorithms to the distributed case.

7. The OSA, OptOSA, DOSA and DOptOSA algorithms have all been fully implemented in $C$ on a SUN/Unix workstation. We report on experiments that we have conducted to determine the trade-offs made when we use OSA vs. OptOSA and DOSA vs. DOptOSA.

\section{Media Objects}

In most multimedia systems, the primitive entities that are being constructed and/or being manipulated are called media objects (or multimedia objects, or sometimes, just plain objects.) However, exactly what constitutes a media-object has often been defined on a case-by-case basis. Intuitively, a media-object could be a video-clip, or a picture, or a text file, or perhaps some complex entity 
constructed out of these simpler entities. In this section, we will provide a formal, mathematical definition of a media-object. As different media-objects have different types of attributes as well as different properties, we classify all media-objects into three types:

1. Static: Intuitively, a static media object is an object that does not change when it is presented. Examples of static media-objects include .gif files and ordinary text files that do not change when presented to the user (though of course they may change as a result of editing by a human).

2. Quasi-Static: A quasi-static media-object is one which may be broken up into a contiguous sequence of chunks of information, each of which is presented to the user sequentially, one after another. However, it is upto the individual viewing the quasi-static object to determine how long to spend in browsing one such chunk of information. A good example of a quasi-static media-object is a postscript file. A user browsing a postscript document through a postscript viewer such as ghostview may move from one page of the postscript file to another at his discretion/leisure.

3. Temporal: A temporal media-object is one which may be broken up into a contiguous sequence of chunks of information, each of which is presented to the user sequentially, one after another. In temporal objects, we asssume that the display time of each "chunk" in the afore-mentioned sequence of contiguous chunks is the same. An example of a temporal media-object is audio, where an analog audio stream may be broken up into 5 millisecond frames for sampling/analysis purposes.

4. Quasi-Temporal: A quasi-temporal media object is similar to a temporal media-object; the only difference is that the display time of different chunks in the afore-mentioned sequence of contiguous chunks may vary. An example of quasi-temporal media-objects could be video where different frames may be of different lengths - this is particularly useful in annotating the video (by hand or otherwise).

Definition 2.1 A media-object o is a 5-tuple consisting of:

1. A data type ds $(o)$ - e.g. the data structure specification may be a .ps file or a .gif file or something else that is completely application-specific.

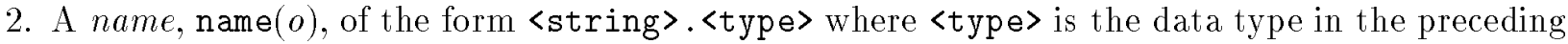
item. For example, if the data type is ps, then the name of such an object may be zzz.ps.

3. An object-type, ot $(o)$, which is either temporal, quasi-temporal, static or quasi-static.

4. An object-characteristic, oc $(o)$, that has the following form:

(a) If a given media-object is of type temporal, then the object characteristic is a pair $(n, \Delta t)$ specifying that the media-object consists of $n$ "chunks" of data, each having duration $\Delta t$.

(b) If the given media-object is of type quasi-temporal, then the object characteristic is a pair $\left(n, \psi_{o}\right)$ where $\psi_{o}$ is a function from $\{1, \ldots, n\} \rightarrow \mathcal{N}$. Intuitively, this means that the media-object $o$ has $n$ "chunks" of data, and that chunk $i$ lasts for time $\psi_{o}(i)$ time units.

(c) If the given media-object is of type quasi-static, then the object characteristic is a pair $(n, \perp)$ denoting that the object has $n$ "chunks" of information where the time taken by each chunk is unpredictable and is determined by the user. 
(d) If the given media-object is of type static, then the object characteristic is of type $(\perp, \perp)$ specifying that the object characteristic is not predictable - neither the number of chunks, nor the time taken in viewing the chunks is predictable and are user-dependent.

5. A component size $(o)$ specifying the size requirements of $o$.

The reader will observe that the above definition is very robust. For example, we may have two different objects

$$
\begin{aligned}
& o_{1}=\left(\mathrm{ds}\left(o_{1}\right), \operatorname{name}\left(o_{1}\right), \operatorname{ot}\left(o_{1}\right), \text { oc }\left(o_{1}\right), \operatorname{size}\left(o_{1}\right)\right) \\
& o_{2}=\left(\mathrm{ds}\left(o_{1}\right), \operatorname{name}\left(o_{1}\right), \operatorname{ot}\left(o_{1}\right), \operatorname{oc}\left(o_{1}\right), \operatorname{size}\left(o_{2}\right)\right)
\end{aligned}
$$

In this example, objects $o_{1}$ and $o_{2}$ are identical except for their size attribute. Such an example may occur, for instance, if a utility such as $\mathrm{xv}$ is used to re-size an image.

Example 2.1 (Text Object) One of the simplest types of media-objects is a textual object. For example, a file of the form a.txt is a textual object having the following properties:

1. Name: a.txt

2. Data Structure Specification: .txt

3. Object Type: static

4. Object-Characteristic: $(\perp, \perp)$,

5. Size: 500 (Kbytes)

Example 2.2 (GIF Object) A slightly more complex media-object is a pictorial object. For example, a file of the form p.gif is a pictorial media-object having the following properties:

1. Name: p.gif

2. Data Structure Specification: .gif

3. Object Type: static

4. Object-Characteristic: $(\perp, \perp)$

5. Size: 580 (Kbytes)

Note that a picture file using a different format (e.g. .tiff) would be defined in a way similar to the above format.

Example 2.3 (Audio Object) Consider, on the other hand, an audio media-object b.avi containing 5000 frames, each of length 2 milliseconds. This media-object is characterized by the following properties:

1. Name: b.avi

2. Data Structure Specification: .avi

3. Object Type: temporal 
4. Object-Characteristic: $(5000,2)$

5. Size: 1 Mbyte

Example 2.4 (Video Object) Consider a slightly more complex situation where we have a video media-object containing 10000 frames, the first 5000 of which are of 2 millisecond duration, the next 2000 of which are of 1 millisecond duration, and the last 3000 of which are of 3 millisecond duration. This media-object is characterized by the following properties:

1. Name: c.avi

2. Data Structure Specification: .avi

3. Object Type: quasi-temporal

4. Object-Characteristic: $(5000, \psi)$ where $\psi$ is the function: $\psi(n)=2$ if $1 \leq n \leq 5000$; 1 if $5001 \leq n \leq 7000$ and 3 if $7000 \leq n \leq 10000$.

5. Size: 3 MBytes.

Certain kinds of objects could be declared in many ways depending upon their intended use. For example, consider a 25 page postscript document (the same comments apply to many other types of documents). This could be declared as a static object (which indicates that the collaborative multimedia system we define will not attempt to automatically have its pages scroll through) or it could be viewed as a temporal object (where each page is displayed for $\Delta t$ time units), or it could be viewed as a quasi-static object where the user scrolls through it at his/her leisure. It is entirely possible that some postscript documents in a collaborative environment are described as temporal objects, while others are defined to be of temporal or quasi-temporal types.

\section{Collaborative Multimedia Systems}

Having defined the concept of a media-object in Section 2, we are now in a position to start work on defining a collaborative multimedia system. Intuitively, such a formal definition should take into account, the following aspects of any collaborative endeavor:

1. Collaborators: First and foremost, we consider a single collaborative effort where there are $k$ collaborators. Each of these collaborators may be located at different locations on the network.

2. Host Capabilities: The site/machine hosting a given collaborator may have a set of capabilities. Such capabilities correspond to the system functionalities available at that host node.

3. Distributed Media Objects: We assume that the purpose of the collaboration is to develop a multimedia-document (a concept to be defined below) that composes together a given set of media-objects. For example, a multimedia-document may be composed of a sequence of video clip $v_{1}$ followed by video clip $v_{2}$ followed by a presentation slide (e.g. .dvi file) followed by an audio file. At any given point in time, a multimedia document may consist of various mediaobjects, located at different sites on the network. These different media-objects may be linked together by various constraints expressing spatial/temporal layout constraints.

In this section, we will provide a formal definition of collaborative multimedia systems. 


\subsection{Simple Collaborative Multimedia Systems}

This section presents the "basic" notion of a collaborative multimedia system. When studying collaboration systems, it is important for the members of the collaboration to be aware of each others capabilities. For the purposes of multimedia collaborations, we will study three types of capabilities:

Definition 3.1 A display capability is a function that maps media-objects to $\{$ true, false .

For example, the Unix utility ghostview may be thought of as a display capability that maps all objects of the form X.ps to true (indicating that it can display them) and all other objects to false indicating that it cannot display them.

Definition 3.2 An edit capability is a function that maps media-objects to \{true, false\}.

For example, if we have a special image editor called ed_tiff to edit .tiff files, then this is an edit-capability that assigns true to all files of the form X.tiff and assigns false to all other objects.

Definition 3.3 A conversion capability is a function that takes as input, a media-object $o$, and returns as output, a media-object $o^{\prime}$.

For example, the standard Unix utility, dvips may be viewed as a conversion capability that converts dvi files to postscript files.

We now define the concept of a simple collaborative multimedia system.

Definition 3.4 A simple collaborative multimedia system (s-COMS for short) consists of:

- an un-directed, weighted graph $G=(V, E, \wp)$ where $V$ is the set of nodes in the graph (representing sites where a member of the collaboration team is located), $E$ refers to the connectivity of the graph, and $\wp: E \rightarrow \mathbf{R}^{+}$specifies the cost of sending a byte of information along an edge in the graph.

- a set $O b j$ of media-objects,

- a function $l o c: O b j \rightarrow V$ specifying where the media-objects are located.

- A set $\mathcal{H C}$ specifying a set of host capabilities - these may not be an exhaustive list of capabilities of participating nodes, but just a list of those capabilities that are of interest for the particular application being developed.

- a function $\mathcal{C A P}: V \rightarrow 2^{\mathcal{H C}}$ specifying what capabilities are available at a given node. (As usual, if $X$ is any set, $2^{X}$ denotes the power set of $X$ ).

Example 3.1 (Motivating Example) Figure 1 shows a diagrammatic representation of a collaborative multimedia system that involves 5 participating entities. The entities are hosts in Seattle, San Diego, Chicago, Ithaca, and College Park. The numbers marked along the edges in the graph shows the weights associated with the edges. A COMS involving the graph of Figure 1 may be described by specifying the values of the different components described in definition 3.4.

1. The undirected weighted graph $G=(V, E, \wp)$ is shown in Figure 1. 


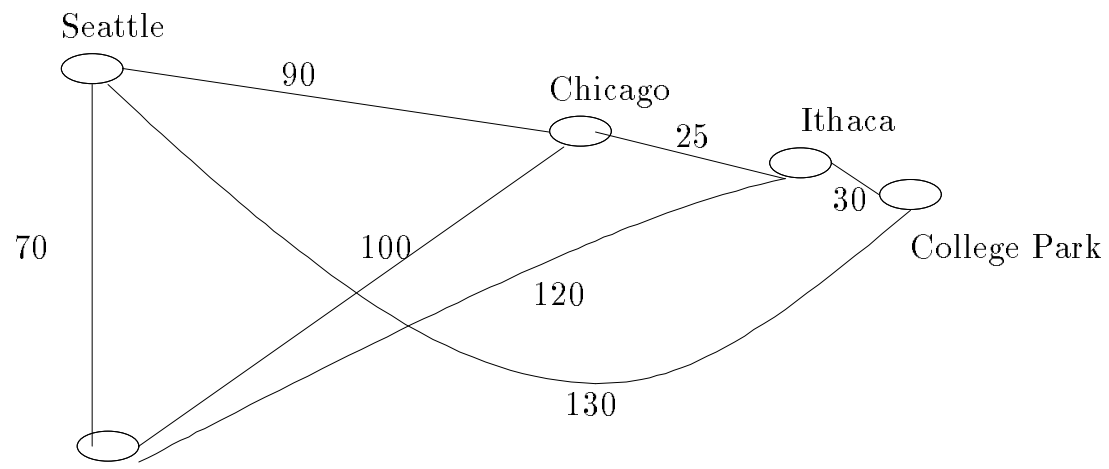

San Diego

Figure 1: Network for COMS of Motivating Example.

2. The set $O b j$ of media-objects involved is shown in the table below:

\begin{tabular}{|c|c|c|c|c|}
\hline o & $\mathrm{d} \mathbf{s}(o)$ & Type & Characteristic & size $(o)$ \\
\hline f1.gif & .gif & static & $(\perp, \perp)$ & 250 \\
\hline f2.txt &.$t x t$ & static & $(\perp, \perp)$ & 45 \\
\hline f3.avi & .avi & temporal & $(5000,10)$ & 975 \\
\hline f4.avi & .avi & quasi-temporal & $\begin{array}{l}\left(4000, \psi_{f 4}\right) \\
\psi_{f 4}(i)= \begin{cases}5 & \text { if } 1 \leq i \leq 3000 \\
8 & \text { if } 3001 \leq i \leq 4000\end{cases} \end{array}$ & 950 \\
\hline f5.tiff & .tiff & static & $(\perp, \perp)$ & 300 \\
\hline f6.vid & .vid & temporal & $(1000,4)$ & 800 \\
\hline f7.vid & .vid & temporal & $(2000,15)$ & 1200 \\
\hline f8.tiff & .tiff & static & $(\perp, \perp)$ & 900 \\
\hline f9.gif & gif & static & $(\perp, \perp)$ & 450 \\
\hline f10.ps & .ps & quasi-static & $(25, \perp)$ & 100 \\
\hline f11.ps & .ps & static & $(\perp, \perp)$ & 140 \\
\hline f12.dvi & dvi & static & $(14, \perp)$ & 100 \\
\hline f13.avi & .avi & quasi-temporal & $\begin{array}{l}\left(2000, \psi_{f 13}\right) \text { where } \\
\psi_{f 13}(i)= \begin{cases}2 & \text { if } 1 \leq i \leq 1000 \\
4 & \text { if } 1001 \leq i \leq 2000\end{cases} \end{array}$ & 1500 \\
\hline
\end{tabular}

3. The function loc specifying the locations of objects is shown in the table below: 


\begin{tabular}{||c|c||}
\hline \hline Object & Location \\
\hline \hline f1.gif & Seattle \\
f2.txt & College Park \\
f3.avi & Ithaca \\
f4.avi & San Diego \\
f5.tiff & Chicago \\
f6.vid & San Diego \\
f7.vid & San Diego \\
f8.tiff & Chicago \\
f9.gif & Seattle \\
f10.ps & College Park \\
f11.ps & College Park \\
f12.dvi & Ithaca \\
f13.avi & Chicago \\
\hline \hline
\end{tabular}

4. The set $\mathcal{H C}$ of host capabilities consists of the following:

\begin{tabular}{||l|l|l||}
\hline \hline Capability Name & Input & Output \\
\hline \hline tiff2bmp & File.tiff & File.bmp \\
\hline tiff2gif & File.tiff & File.gif \\
\hline gif2tiff & File.gif & File.tiff \\
\hline dvips & File.dvi & File.ps \\
\hline capture_image & (File.vid,integer) & File.gif \\
\hline audio2text & File.avi & File.txt \\
\hline ghostview & File.ps & display(file.ps) \\
\hline xtex & File.dvi & display(file.dvi) \\
\hline xv1 & File.tiff & display(File.tiff) \\
\hline xv2 & File.gif & display(File.gif) \\
\hline showvid & File.vid & display(File.vid) \\
\hline showavi & File.avi & display(File.avi) \\
\hline ed_tiff & File.tiff & File.tiff \\
\hline \hline
\end{tabular}

5. The mapping $\mathcal{C} \mathcal{A P}$ is defined as shown below:

\begin{tabular}{||l|l||}
\hline \hline$V$ & $\mathcal{C A} \mathcal{A}(V)$ \\
\hline \hline Seattle & $\{$ xv1,dvips, xtex, gif2tiff,tiff2bmp $\}$ \\
\hline San Diego & $\{$ capture_image, audio2text, showavi $\}$ \\
\hline Chicago & $\{$ capture_image,gif2tiff,dvips, showavi $\}$ \\
\hline Ithaca & $\{$ xv1, xv2, gif2tiff,tiff2gif,ghostview, xtex $\}$ \\
\hline College Park & $\{$ ed_tiff,tiff2gif, audio2text,ghostview $\}$ \\
\hline \hline
\end{tabular}

\subsection{Collaboration in an s-COMS: An Informal Description}

Collaboration in an s-COMS is accomplished through read-write requests made by the different collaborators involved. For example, consider the example s-COMS given in Section 3.1. The individual 
located in College Park may wish to work on the image file $\mathbf{f} 1 . g i f$ located in Seattle. For the time being, we assume that all partners participating in an s-COMS have full read/write access to all objects in $O b j$. What happens when the College Park collaborator wishes to work on file f1.gif ?

Checkout: First of all, the College Park collaborator must send a request to a server in Seattle requesting the desired files. The server in Seattle must try to send the documents to the College Park collaborator, keeping in mind the fact that the person in College Park must be able to edit the .gif file. However, we know that the College Park collaborator cannot edit .gif files as he has no .gif editor. Therefore either the server in Seattle or the client in College Park must find a "loop" in the graph/network such that:

- The loop is of the form $\ell_{1}, \ldots, \ell_{i}, \ldots, \ell_{n}$ where $\ell_{1}=$ Seattle $=\ell_{n}$ and $\ell_{i}=$ College Park.

- There is some $j, 1 \leq j \leq i$ such that $\mathcal{H C}\left(\ell_{j}\right)$ achieves the goal of converting the .gif file to an image file that can be edited using the a.tiff editor that the College Park site has. The conversion does not need to be done on a single node on the path. Instead, each node can help to the process of conversion by performing subconversions.

- Once the College Park collaborator has completed his/her work on the .tiff file, he must return the file to Seattle in .gif format - the format the Seattle site expects the file to be returned in. This means that there must be some $k, i \leq k \leq n$ such that $\mathcal{H C}\left(\ell_{k}\right)$ achieves the goal of converting the .tiff file to a .gif file.

- Finally, the total network cost should be minimized, i.e. the cost of sending the file across the different links on the network must be minimized.

In general, it is preferable if the client specifies the loop to be used - as the server may be servicing multiple clients, passing this responsibility onto the server may lead to an unacceptable load on the server. Furthermore, a server may decline to compute such a path, and hence, the client should be responsible for specifying the path by which the desired object is routed.

If we examine the example in Section 3.1, then the desired loop is:

$$
\text { Seattle } \rightarrow \text { CollegePark } \rightarrow \text { Ithaca } \rightarrow \text { CollegePark } \rightarrow \text { Seattle }
$$

This means that when Seattle is initially requested for the .gif file by College Park, it sends it directly to College Park who passes in to Ithaca which in turn converts it (using gif2tiff) to tiff format and passes it back to College Park who edits the tiff file using ed_tiff and then converts it to .gif format at College Park itself using tiff2gif and sends the result back directly to Seattle. If we assume that the cost is independent of the size of the objects, then the total cost of this operation is

$$
(130+30+30+130) \times 250=80000 .
$$

The reader may easily verify that this total cost is the cheapest possible total cost possible, even though other loops may be used to satisfy the same objective.

\subsection{Collaboration in an s-COMS: A Formal Description}

Consider an s-COMS $\Gamma=(G, O b j$, loc, $\mathcal{H C}, \mathcal{C} \mathcal{A P})$ where $G=(V, E)$. Suppose $N \in V$ is a node in the network and $o$ is an object that we wish to create from existing objects. 
Definition 3.5 A capability $c$ may be applied to object $o$ just in case $o$ is of the input-type of capability $c$. If $c$ is a conversion capability, then the result of applying capability $c$ to object $o$ is $c\left(o^{\prime}\right)$. This is denoted by

$$
o \stackrel{c}{\longrightarrow} o^{\prime}
$$

\subsubsection{Synthesizing Objects within a Node}

We will first consider the case when objects are constructed within a single node, using only the objects and capabilities contained within that node. It will turn out that once we know how to synthesize objects within a given node, then we may use this solution to solve the problem of synthesizing objects in a network of nodes.

Definition 3.6 An object $\boldsymbol{o}_{m}$ may be synthesized entirely within node $N$ just in case there is a sequence of objects $o_{1}, \ldots, o_{m}$ such that:

1. $N \in \operatorname{loc}\left(o_{1}\right)$, i.e. object $o_{1}$ is available to node $N$ and

2. For all $1<i \leq m$, there is a conversion capability $c_{i-1} \in \mathcal{H C}(N)$ such that

$$
o_{i-1} \stackrel{c_{i-1}}{\longrightarrow} o_{i}
$$

holds.

We will call

$$
o_{1} \stackrel{c_{1}}{\longrightarrow} o_{2} \stackrel{c_{2}}{\longrightarrow} o_{3} \stackrel{c_{3}}{\longrightarrow} \cdots o_{m-1} \stackrel{c_{m-1}}{\longrightarrow} o_{m}
$$

a synthesis sequence for object $o_{m}$ within node $N$.

Definition 3.7 An object o may be displayed (resp. edited) entirely within node $N$ if there is a synthesis sequence for object $o$ within node $N$ and there is a display (resp. edit capability) capability, $\mathcal{D} \in \mathcal{H C}(N)$ such that $\mathcal{D}(o)=$ true.

We now present an algorithm that solves the following problem:

Object Synthesis Problem. Given a node $N$, and an object $o$, determine if $o$ can be synthesized entirely within node $N$. If yes, return a synthesis sequence for object $o$ within node $N$.

\section{Algorithm 1 (Object Synthesis Algorithm (OSA))}

\section{Input:}

- A COMS $\Gamma=(G, O b j, l o c, \mathcal{H C}, \mathcal{C} \mathcal{A P})$ where $G=(V, E)$

- A node $N$ in $V$ and

- An object $o$ to be synthesized entirely within node $N$.

Tried: A global set variable initialized to $\emptyset$.

1. Let Name(o) be of the form str.type.

2. Let $X=\{(c$, Ob.type 1$) \mid c$ is a conversion capability in $\mathcal{H C}(N)$ and $\operatorname{Name}(c($ Ob.type 1$))=$ str.type $\}$. (* Note that $O b$ may not necessarily be such that $N \in \operatorname{loc}(O b)^{*}$ ). 
3. if $\{(c$, Ob.type 1$) \mid(c$, Ob.type 1$) \in X \&(c$, Ob.type $) \notin$ Tried $\}=\emptyset$ then return failure and halt.

4. Otherwise (* i.e. $X$ still has something in it that can be processed *)

5. if there exists a $(c, O b) \in X$ such that $(c, O b) \notin$ Tried and such that $N \in \operatorname{loc}(O b)$ then return

$$
\text { SOL }=\text { Ob.type } 1 \stackrel{c}{\rightarrow} \text { str.type }
$$

and halt.

6. Otherwise, non-deterministically select a pair $(c, O b) \in X$.

7. Set $X$ to $X-\{(c, O b)\}$ and set Tried to Tried $\cup\{(c, O b)\}$.

8. If the call $\operatorname{OSA}(\Gamma, N, O b)$ succeeds and returns $S O L 1$, then

$$
\text { SOL }=S O L 1 \stackrel{c}{\longrightarrow} \text { str.type. }
$$

Return SOL. Halt with success.

9. Otherwise, goto Step 3.

To see how the above algorithm works, let us return to the motivating example COMS discussed in Section 3.1.

Example 3.2 Suppose we wish to synthesize the object named f1.bmp in Seattle. In other words, for whatever reason, the site at Seattle wishes to obtain a bitmapped version of $f 1$.gif. In this case, we call the OSA algorithm with the example COMS, the node Seattle and the object $f 1 . b m p$ that we wish to synthesize.

- In step 2 of the OSA algorithm, we set

$$
X=\{(\text { tiff2bmp, f1.tiff })\} .
$$

This indicates that:

- One way to synthesize $\mathbf{f} 1$. bmp is by first synthesizing a file $\mathbf{f} 1$.tiff and then by applying the operator tiff 2 bmp to convert it into a bitmapped file.

- The test in Step 3 succeeds, but that in Step 5 fails, and control passes to Step 6. The only triple that can be selected is (tiff $2 \mathrm{bmp}, \mathrm{f} 1$.tiff), so it is selected and in Step 7, we reset $X$ to $\emptyset$ and Tried is set to $\{($ tiff 2 bmp, f1.tiff $)\}$.

- In step 8, we call the OSA algorithm recursively with a request to synthesize $\mathrm{f} 1$. tiff.

- This latter request succeeds immediately via step 5 , leading to $S O L 1=$ f1.gif $\stackrel{\text { gif2tiff }}{\longrightarrow}$ f1.tiff.

- In step 8 of the original call, we now return

$$
S O L=\text { f1.gif } \stackrel{\text { gif2tiff }}{\longrightarrow} \text { f1.tiff } \stackrel{\text { tiff2bmp }}{\longrightarrow} \text { f1.bmp. }
$$

- The algorithm now halts.

Example 3.3 A slightly more egregious example shows what can happen when a node contains "reversible" actions. For instance, suppose $\Gamma$ is a COMS containing just one node having the object o1.f $m t 1$ and having two conversion functions: 
1. $c_{1}$ that converts $X$.fmt 1 to $X$.fmt2;

2. $c_{2}$ that converts $X . f m t 2$ to $X . f m t 1$;

Suppose we wish to create an object $o 2 . f m t 2$. The OSA algorithm terminates because the global variable Tried in it is initially empty; after executing step $3, X=\left\{\left(c_{1}, o 2 . f m t 1\right)\right\}$; however, after step $7, X=\emptyset$ and Tried $=\left\{\left(c_{1}, o 2 . f m t 1\right)\right\}$; in step 8, we recursively call the OSA algorithm with arguments $(\Gamma, N, o 2 . f m t 1)$. In step 3 of the recursive call, $X=\left\{\left(c_{2}, o 2 . f m t 2\right)\right\}$; in Step $7, X=\emptyset$ and Tried $=\left\{\left(c_{1}, o 2 . f m t 1\right),\left(c_{2}, o 2 . f m t 2\right)\right\}$. In Step 8, we recursively call the OSA algorithm with arguments ( $\Gamma, N, o 2 . f m t 2)$. In Step 3 of the recursive call, we return with failure, cuasing the initial invocation of the OSA algorithm to terminate with failure.

The following result tells us that the OSA algorithm is sound and complete, and terminates in lineartime (proofs have been omitted for brevity).

Theorem 3.1 (Soundness and Completeness of the OSA Algorithm) Suppose $\Gamma=(G, O b j, l o c$, $\mathcal{H C}, \mathcal{C} \mathcal{A P})$ is a COMS, $G=(V, E), N$ is a node in $V$, and $o$ is an object we wish to synthesize.

1. If $S S$ is a synthesis sequence for object $o$ within node $N$, then there exists a way of selecting pairs in Step 6 of the OSA algorithm such that the OSA algorithm terminates with success and returns $S O L=S S$.

2. If the OSA algorithm terminates with failure, then there is no synthesis sequence for object $o$ within node $N$.

3. If the OSA algorithm terminates with success and returns $S O L$, then $S O L$ is a synthesis sequence for object $o$ within node $N$.

4. The OSA algorithm is guaranteed to terminate in time $O\left(\operatorname{card}(\mathcal{H C}(N)) \times \operatorname{card}\left(O b j_{N}\right)\right)$ where $O b j_{N}=\{o \mid N \in \operatorname{loc}(o)\}$.

\subsubsection{Optimally Synthesizing Objects within a Node}

The OSA algorithm assumes that any way of synthesizing an object is acceptable. However, in practice, different ways of synthesizing objects may lead to very different results. For example, a synthesis sequence $S S_{1}$ to synthesize an object may involve invoking various expensive conversion methods. In contrast, a different synthesis sequence $S S_{2}$ may achieve the desired synthesis in a "much cheaper" way. In this subsection, we will define the "cost" of a synthesis sequence and then develop a technique called the OptOSA technique that is always guaranteed to optimally synthesize objects within a given node.

Let us suppose that each conversion capability $c_{i}$ has an associated cost rate, cost_rate $\left(c_{i}\right)$, and an associated size ratio, size_ratio $\left(c_{i}\right)$. Intuitively, if the cost rate of $c_{i}$ is 24 , then this means that the cost of converting an object $o$ of size size $(o)$ bytes is $24 \times \operatorname{size}(o)$. Similarly, if the the size ratio of $c_{i}$ is 1.6 , then this means that the size of the object $c_{i}(o)$ is 1.6 times the size of object $o$. Therefore, if

$$
S S=o_{1} \stackrel{c_{1}}{\longrightarrow} o_{2} \stackrel{c_{2}}{\longrightarrow} o_{3} \stackrel{c_{3}}{\longrightarrow} \cdots o_{m-1} \stackrel{c_{m-1}}{\longrightarrow} o_{m}
$$


is a synthesis sequence for object $o$ within node $N$, then the total cost of synthesizing $o$ entirely within node $N$ is given by:

$$
\operatorname{Tot} \operatorname{Cost}(\mathrm{SS})=\sum_{i=1}^{m-1} \operatorname{cost\_ rate}\left(c_{i}\right) \times \operatorname{size}\left(o_{i}\right) .
$$

As $\operatorname{size}\left(o_{i}\right)=\operatorname{size}\left(o_{i-1}\right) \times \operatorname{size} \_r a t i o\left(c_{i-1}\right)$ for $i>1$, it follows that

$$
\operatorname{size}\left(o_{i}\right)=\operatorname{size}\left(o_{1}\right) \times \prod_{j=1}^{i-1} \operatorname{size} \_ \text {ratio }\left(c_{j}\right) \text { when } i>1
$$

Thus,

$$
\operatorname{Tot} \operatorname{Cost}(\mathrm{SS})=\sum_{i=1}^{m-1}\left(\operatorname{cost\_ rate}\left(c_{i}\right) \times\left(\operatorname{size}\left(o_{1}\right) \times \prod_{j=1}^{i-1} \operatorname{size\_ ratio}\left(c_{j}\right)\right)\right) .
$$

When attempting to synthesize an object entirely within a given node, we would like to find a synthesis sequence $S S$ that has the least possible total cost TotCost(SS). Obviously, we would like to do this without explicitly constructing all possible synthesis sequences for object $o$ within node $N$.

Optimal Object Synthesis Problem. Given a node $N$, and an object $o$, find the minimal cost (if one exists) synthesis sequence for object $o$ within node $N$.

Before developing an algorithm to efficiently compute optimal ways of synthesizing objects, we present a couple of examples to illustrate the basic ideas.

Example 3.4 Suppose we consider a very simple COMS $\Gamma$ containing a node $N$ that has five conversion functions $c_{1}, \ldots, c_{5}$ described below.

\begin{tabular}{||l|l|l|l||}
\hline \hline Capability Name & Input & Output & Cost Rate \\
\hline \hline$c_{1}$ & X.f1 & X.f2 & 10 \\
\hline$c_{2}$ & X.f2 & X.f3 & 3 \\
\hline$c_{3}$ & X.f2 & X.f5 & 20 \\
\hline$c_{4}$ & X.f3 & X.f4 & 4 \\
\hline$c_{5}$ & X.f4 & X.f5 & 7 \\
\hline \hline
\end{tabular}

Suppose node $N$ has one object named o.f 1 of size 50 Kbytes and suppose we wish to synthesize the object $o . f 5$. Furthermore suppose all of transformations $c_{1}, \ldots, c_{5}$ are size-invariant, i.e. the sizes of the objects do not change when these conversions are applied to them. In other words, size_ratio $\left(c_{i}\right)=1$ for $i=1, \ldots, 5$. There are two synthesis sequences that accomplish the desired goal. They are:

$$
\begin{gathered}
S S_{1}: \quad \text { o.f } 1 \stackrel{c_{1}}{\longrightarrow} \text { o.f } 2 \stackrel{c_{2}}{\longrightarrow} \text { o.f } 3 \stackrel{c_{4}}{\longrightarrow} \text { o.f } f \stackrel{c_{5}}{\longrightarrow} \text { o.f } 5 \\
S S_{2}: \quad \text { a.f } 1 \stackrel{c_{1}}{\longrightarrow} \text { a.f } 2 \stackrel{c_{3}}{\longrightarrow} \text { a.f5. }
\end{gathered}
$$

Even though $S S_{1}$ is a longer sequence (in terms of the number of conversions that must be performed), it turns out, in this example, that the total cost of $S S_{1}$ is less than the total cost of $S S_{2}$.

$$
\begin{gathered}
\operatorname{Tot} \operatorname{Cost}\left(S S_{1}\right)=(10 \times 50)+(3 \times 50)+(4 \times 50)+(7 \times 50)=1200 . \\
\operatorname{Tot} \operatorname{Cost}\left(S S_{2}\right)=(10 \times 50)+(20 \times 50)=1500 .
\end{gathered}
$$


Example 3.5 Suppose we consider another very simple COMS $\Gamma$ containing a node $N$ that has four conversion functions $c_{1}, \ldots, c_{4}$ described below. Node $N$ has one object named $o . f 1$ of size $s$ Kbytes.

\begin{tabular}{||l|l|l|l|l||}
\hline \hline Capability Name & Input & Output & Cost Rate & Size Ratio \\
\hline \hline$c_{1}$ & X.f1 & X.f2 & 5 & 2 \\
\hline$c_{2}$ & X.f1 & X.f3 & 9 & 3 \\
\hline$c_{3}$ & X.f2 & X.f4 & 8 & 1 \\
\hline$c_{4}$ & X.f3 & X.f4 & 3 & 1 \\
\hline \hline
\end{tabular}

Suppose we are interested in synthesizing the object o.f 4 . There are two synthesis sequences that accomplish the desired goal. They are:

$$
S S_{1}: \quad \text { o.f } 1 \stackrel{c_{1}}{\rightarrow} \text { o.f } 2 \stackrel{c_{3}}{\longrightarrow} \text { o.f } 4
$$

and

$$
\begin{aligned}
& S S_{2}: \quad \text { o.f } 1 \stackrel{c_{2}}{\longrightarrow} \text { o.f } 3 \stackrel{c_{4}}{\longrightarrow} \text { o.f } 4 . \\
& \operatorname{Tot} \operatorname{Cost}\left(S S_{1}\right)=5 s+16 s=21 s . \\
& \operatorname{Tot} \operatorname{Cost}\left(S S_{2}\right)=9 s+9 s=18 s .
\end{aligned}
$$

Therefore, $S S_{2}$ is a cheaper way of synthesizing object $o . f 4$.

We are now ready to present the OptOSA technique for finding optimal ways of synthesizing objects. The OptOSA technique for finding optimal ways of synthesizing objects attempts to first construct an object bottom-up by iteratively computing a function $T$ defined below.

\section{Definition 3.8 (Operator $T$ )}

\section{Input:}

- A COMS $\Gamma=(G, O b j, l o c, \mathcal{H C}, \mathcal{C} \mathcal{A P})$ where $G=(V, E)$

- A node $N$ in $V$ and

- An object $o$ of the form X.type that we wish to synthesize in an optimal manner.

Output: An optimal synthesis sequence for object $o$ entirely within node $N$.

1. $T^{0}($ X.type $)=\{(X . t, \operatorname{size}(X . t), 0, X . t) \mid N \in \operatorname{loc}(X . t)\}$.

2. $T^{i+1}(X$. type $)=T^{i}(X$. type $) \cup\left\{(X . t, S, C, S e q) \mid\right.$ there is a quadruple $\left(X . t^{\prime}, S 1, C 1, S e q 1\right) \in$ $T^{i}$ (X.type $)$ and there exists a $c \in \mathcal{H C}(N)$ such that $c\left(X . t^{\prime}\right)=X$.t and $C=C 1+$ cost_rate $(c) \times S 1$ and $S=$ size_ratio $(c) \times S 1$ and $S e q=S e q 1 \stackrel{c}{\longrightarrow} X . t\}$.

To see how the above definition works, consider the following example: 
Example 3.6 Suppose we wish to synthesize the object a.tiff within a node that has the following capabilities:

$$
\text { dvi2ps, ps2tiff, dvi2tiff, dvi2bmp, bmp2tiff. }
$$

As is common, $\mathrm{x} 2 \mathrm{y}$ indicates a conversion capability that converts files of type $\mathrm{x}$ to one of type $\mathrm{y}$. Furthermore, suppose that the file a.dvi of size (1000 bytes) is available within node $N$. In addition, size_ratio $\left(c_{i}\right)$ and cost_rate $\left(c_{i}\right)$ are given by:

size_ratio(dvi2ps $)=3$; size_ratio(ps2tiff $)=1.4$; size_ratio(dvi2tiff $)=10$; size_ratio(dvi2bmp $)=14$; size_ratio(bmp2tiff $)$ $=1.2$;

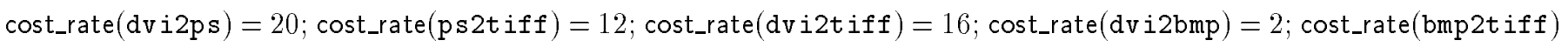
$=9$;

There are three synthesis sequences that can be used to synthesize object a.tiff. These sequences are:

$$
\begin{gathered}
\mathrm{SS}_{1}: \text { a.dvi } \stackrel{\text { dvi2ps }}{\longrightarrow} \text { a.ps } \stackrel{\text { ps2tiff }}{\longrightarrow} \text { a.tiff. } \\
\mathrm{SS}_{2}: \text { a.dvi } \stackrel{\text { dvi2tiff }}{\longrightarrow} \text { a.tiff. } \\
\mathrm{SS}_{3}: \text { a.dvi } \stackrel{\text { dvi2bmp }}{\longrightarrow} \text { a.bmp } \stackrel{\text { bmp2tiff }}{\longrightarrow} \text { a.tiff. }
\end{gathered}
$$

The operator $T$ works in the following way:

- $T^{0}($ a.tiff $)=\{($ a.dvi, 1000,0, a.dvi $)\}$.

- $T^{1}($ a.tiff $)=T^{0}($ a.tiff $) \cup\{$

(a.ps, 3000, 20000, a.dvi $\stackrel{\text { dvi2ps }}{\longrightarrow}$ a.ps),

(a.tiff, 10000,16000, a.dvi $\stackrel{\text { dvi2tiff }}{\longrightarrow}$ a.tiff $)^{\mathrm{SS}_{1}}$,

(a.bmp, 14000, 2000, a.dvi $\stackrel{\text { dvi2bmp }}{\longrightarrow}$ a.bmp)\}.

- $T^{2}($ a.tiff $)=T^{1}($ a.tiff $) \cup\{$

$$
\begin{aligned}
& \text { (a.tiff, 4200,56000, a.dvi } \stackrel{\text { dvi2ps }}{\longrightarrow} \text { a.ps } \stackrel{\text { ps2tiff }}{\longrightarrow} \text { a.tiff })^{\mathrm{SS}_{2}} . \\
& (\text { a.tiff, 16800, 128000, a.dvi } \stackrel{\text { dvi2bmp }}{\longrightarrow} \text { a.bmp } \stackrel{\text { bmptiff }}{\longrightarrow} \text { a.tiff })^{\mathrm{SS}_{3}} .
\end{aligned}
$$

It is easy to see that $T^{2}$ (a.tiff) $=T^{3}$ (a.tiff) and hence, $T^{2}$ (a.tiff) is a fixpoint of the operator $T$. Each of the marked quadruples in this fixpoint encodes one of the sequences $S S_{1}, S S_{2}, S S_{3}$ together with the cost of that synthesis sequence. The reader will easily observe that the cheapest cost sequence is $S S_{1}$ whose total cost is only 16000 . Figure 2 shows a diagrammatic rendering of the sequences involved.

The reader will notice that each and every possible synthesis sequence for the object a.tiff is present in the fixpoint $T^{2}$ (a.tiff) in the above example, and furthermore, that this fixpoint enumerates each and every "path" between a.dvi and a.tiff in Figure 2. The OptOSA algorithm will in many cases, never explore many of these paths by optimizing the computation of the fixpoint of $T^{2}$ (a.tiff) so as to eliminate paths that are not likely to lead to a low cost. However, before developing the OptOSA algorithm, we present some elementary properties of the $T$ operator.

Lemma 3.1 (Properties of $T$ Operator) Consider a COMS $\Gamma$, a node $N$ in $\Gamma$, and an object $o$ of the form X.type that we wish to synthesize within node $N$. For all $j$, if $T^{j}$ (X.type) contains a quadruple of the form $\left(o^{\prime}\right.$, size $\left.\left(o^{\prime}\right), C^{\prime}, S S^{\prime}\right)$, then there is a synthesis sequence $S S^{\prime}$ of object $o^{\prime}$ entirely within node $N$. 


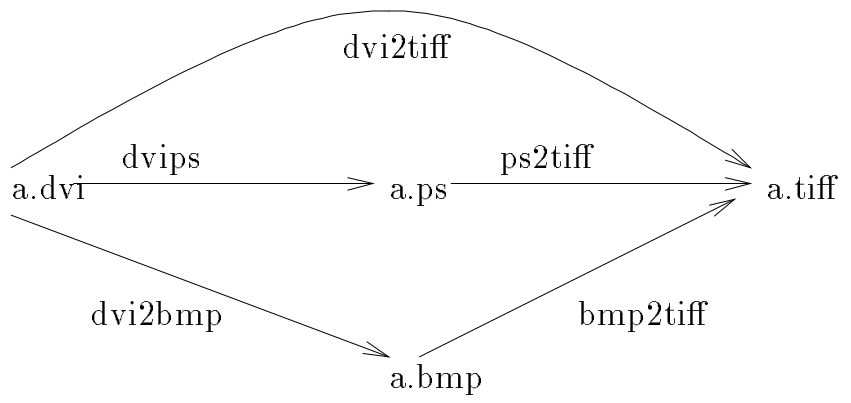

Figure 2: Synthesis Sequences for Example

The main problem with the above lemma is that it computes all synthesis sequences for the object $o$. However, in practice, we would like to compute an optimal (i.e. least cost) synthesis sequence without computing all such sequences. We are now ready to define the OptOSA technique which efficiently computes optimal synthesis sequences.

\section{Algorithm 2 (Optimal Object Synthesis Algorithm (OptOSA))}

\section{Input:}

- A COMS $\Gamma=(G, O b j, l o c, \mathcal{H C}, \mathcal{C} \mathcal{A P})$ where $G=(V, E)$

- A node $N$ in $V$ and

- An object $o=$ a.type to be synthesized in an optimal manner.

Output: An optimal synthesis sequence for object $o$ entirely within node $N$.

1. Our algorithm uses a special node structure that has the following fields: name, size, cost, seq, overallcost, next - the first four of these fields refer to the four components of the quadruples manipulated in Definition 3.8. overallcost refers to the total cost of the sequence associated with a node. In other words, if a node has $S S$ in its seq field, then the overallcost field contains the value TotCost defined earlier. The next field is a pointer to another node of the same type.

2. SOL $:=N I L$.

3. Compute $T^{0}$ (a.type).

4. For each quadruple of the form (a.t, size(a.t), 0, a.t) in $T^{0}$ (a.type), create a node $V$ having

$$
\begin{gathered}
V . \text { name }=a . t ; V \cdot \operatorname{size}=\operatorname{size}(a . t) ; \\
V . \cos t=0 ; V . \text { seq }=a . t ; V \text {.overallcost }=0 .
\end{gathered}
$$

Let $X$ be a linked list consisting of all these nodes.

(* We will always assume that $X$ is sorted in ascending order according to the overallcost field. As initially all these nodes have their overallcost field set to 0 , this means that when this step is first executed, any ordering will suffice. ${ }^{*}$ )

5. if $X=N U L L$ then halt with failure. 
6. else

(a) i. Cur $:=\operatorname{head}(X) ; X:=\operatorname{tail}(X)$;

ii. if $C$ ur.name $=$ a.type then return $C$ ur.seq and halt.

iii. Cand $:=\left\{\left(c, o^{\prime}\right) \mid c \in \mathcal{H C}(N)\right.$ and $c(C u r$.name $)=o^{\prime}$ for and $\left.N \in \operatorname{loc}\left(o^{\prime}\right)\right\}$;

iv. For each $\left(c, o^{\prime}\right) \in$ Cand create a node $V_{c, o^{\prime}}$ with fields:

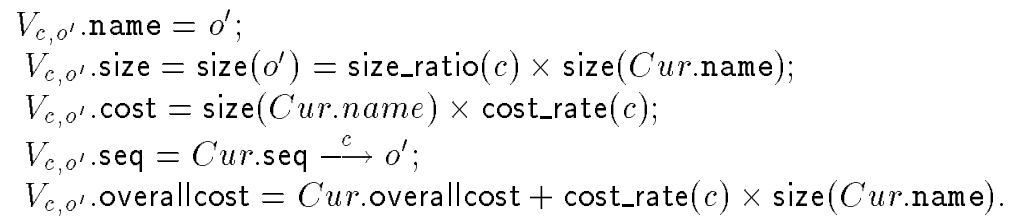

if there is not a node $M$ in $V_{c, o^{\prime}}$.seq such that $V_{c, o^{\prime}}$.name $=M$.name and $V_{c, o^{\prime}}$.size $\geq$ $M$.size then insert $V_{c, o^{\prime}}$ into the list $X$, keeping $X$ in sorted order w.r.t. the totcost field.

v. Goto Step 5 .

To illustrate why the OptOSA algorithm works much more efficiently than the OSA algorithm, let us return to Example 3.6.

Example 3.7 The reader may already have noticed that $T^{1}$ (a.tiff) contains a synthesis sequence which is captured by the quadruple:

$$
\text { (a.tiff, } 10000,16000, \text { a.dvi } \stackrel{\text { dvi2tiff }}{\longrightarrow} \text { a.tiff). }
$$

This quadruple says that the object a.tiff may be synthesized by using the synthesis sequence

$$
\text { (a.dvi } \stackrel{\text { dvi2tiff }}{\longrightarrow} \text { a.tiff) }
$$

at a total cost of 16,000 . Notice that in $T^{1}$ (a.tiff), we also have the quadruple

$$
\text { (a.ps, 3000, 20000, a.dvi } \stackrel{\text { dvips }}{\longrightarrow} \text { a.ps) }
$$

which only goes part of the way towards computing a synthesis sequence for a.tiff, but which has already incurred a higher cost, viz. 20,000. Furthermore, in the definition of the $T$ operator, this quadruple leads to the generation of the new quadruple

$$
\text { (a.tiff, 4200,56000, a.dvi } \stackrel{\text { dvips }}{\longrightarrow} \text { a.ps } \stackrel{\text { ps2tiff }}{\longrightarrow} \text { a.tiff) }
$$

in $T^{2}$ (a.tiff) even though there is already a better solution. The OptOSA algorithm would eliminate such redundant possibilities. To see how, let us now apply the OptOSA algorithm to the case of Example 3.6. Here is what happens:

- Initially, $T^{0}($ a.tiff $)=\{($ a.dvi, 1000, 0, a.dvi $)\} . X$ points to a list of one node containign the quadruple ( (a.dvi,1000,0,a.dvi). 
- Step 6(a) of the OptOSA algorithm now leads to the following quadruples stored in the order listed below (the order is significant).

$$
\begin{aligned}
& \text { (a.bmp, 14000, 2000, a.dvi } \stackrel{\text { dvi2bmpf }}{\longrightarrow} \text { a.bmp). } \\
& \text { (a.tiff, 10000, 16000, a.dvi } \stackrel{\text { dvi2tiff }}{\longrightarrow} \text { a.tiff), } \\
& \text { (a.ps, } 3000,20000, \text { a.dvi } \stackrel{\text { dvips }}{\longrightarrow} \text { a.ps). }
\end{aligned}
$$

- The first element in this list is the quadruple

$$
\text { (a.bmp, 14000, 2000, a.dvi } \stackrel{\text { dvi } 2 \text { bmp }}{\longrightarrow} \text { a.bmp). }
$$

Note that at this stage, it is still possible that there is a cheap synthesis sequence which first converts a.dvi to a.tiff via an intermediate conversion to a.bmp. That is why the solution of cost 16000 in the above list of quadruples is not returned immediately.

- At the next stage, $X$ now points to the ordered list:

$$
\begin{aligned}
& \text { (a.tiff, 10000, 16000, a.dvi } \stackrel{\text { dvi2tiff }}{\longrightarrow} \text { a.tiff }), \\
& \text { (a.ps, 3000, 20000, a.dvi } \stackrel{\text { dvips }}{\longrightarrow} \text { a.ps). } \\
& \text { (a.tiff, 16800, 128000, a.dvi } \stackrel{\text { dvi2bmp }}{\longrightarrow} \text { a.bmp } \stackrel{\text { bmp2tiff }}{\longrightarrow} \text { a.tiff). }
\end{aligned}
$$

- In the next stage, the first element of this list, viz. the quadruple

$$
\text { (a.tiff, } 10000,16000, \text { a.dvi } \stackrel{\text { dvi2tiff }}{\longrightarrow} \text { a.tiff) }
$$

is returned as the optimal solution.

The reader will notice that many quadruples listed in Example 3.6 never occur in this computation, because they have been discarded by the OptOSA algorithm, thus leading to highly improved performance (Section 4 reports on experimental results).

Theorem 3.2 (Soundness and Completeness of the OptOSA Algorithm) Suppose $\Gamma=(G, O b j, l o c$, $\mathcal{H C}, \mathcal{C} \mathcal{A P})$ is a COMS, $G=(V, E), N$ is a node in $V$, and $o$ is an object we wish to synthesize. Then:

1. If the OptOSA algorithm returns a synthesis sequence, then that synthesis sequence is an optimal synthesis sequence for object $o$ within node $N$.

2. The OptOSA algorithm returns with failure iff there is no synthesis sequence for object $o$ in node $N$.

3. If $\left\{S S_{1}, \ldots, S S_{n}\right\}, n \geq 1$, is the set of all optimal synthesis sequences for $N$, then the OptOSA algorithm is guaranteed to return some $S S_{i}$.

Theorem 3.2 is extremely important for a number of reasons. First and foremost, the theorem says that the OptOSA algorithm correctly finds the optimal synthesis sequence for any object that we wish to construct. Furthermore, it says that the OptOSA algorithm always finds the optimal solution first, without finding any other solutions at all. In other words, the search strategy used by OptOSA in looking for an optimal synthesis sequence is perfect - the first synthesis sequence it comes up with is guaranteed to be the best one - hence, there is no need to evaluate multiple alternative sequences and pick the best one. 


\subsubsection{Optimal Object Synthesis with Quality Constraints}

One of the key problems that has not been discussed in the preceding sections is the issue of quality. Many transformations may cause the size of a media-object to decrease, yet these transformations may not preserve the quality of the object. For example, utilities like xv in the Unix platform allow us to re-size images (like those in tiff and gif files). In such cases, we need to know whether the transformations affect the quality of the image - for example, when we reduce an image to $50 \%$ of its original size, this is accompanied by a concomitant loss of quality. Often users not only wish to synthesize objects, but they wish to synthesize objects with a certain quality measure.

In this section, we will study the problem of synthesizing objects in the presence of quality constraints. Even more important is the fact that in many cases, a user may wish to simultaneously achieve two objectives:

(Objective 1) minimize $S S$ overallcost $(S S)$ - i.e. minimize the cost of synthesizing the object, and

(Objective 2) maximize quality.

However, it is entirely possible that these two goals may conflict with one another, and we will study ways to deal with such conflicts.

Consider an s-COMS $\Gamma=(G, O b j, l o c, \mathcal{H C}, \mathcal{C} \mathcal{A P})$ where $G=(V, E)$. A quality-assessment is a function $\mathrm{QA}: O b j \rightarrow \mathbf{R}^{+}$where $\mathbf{R}^{+}$is the set of non-negative real numbers. Intuitively, $\mathbf{Q A}\left(o_{1}\right)=300$ says that the quality of object $o_{1}$ is deemed to be 300 w.r.t. some scale. Thus, if $\mathrm{QA}\left(o_{2}\right)$ were 200 , then $o_{1}$ would be a "better" quality object than $o_{1}$.

A quality function is a map $Q F: \mathcal{H C} \rightarrow[0.0,1.0]$. Intuitively, $Q F($ tiff2gif $)=0.9$ says that when a tiff-image is converted to gif-format, the resulting gif image is only $90 \%$ as good as the original.

Suppose $S S$ is a synthesis sequence for an object $o_{m}$, and suppose $S S$ has the form

$$
o_{1} \stackrel{c_{1}}{\rightarrow} o_{2} \stackrel{c_{2}}{\longrightarrow} o_{3} \stackrel{c_{3}}{\longrightarrow} \cdots o_{m-1} \stackrel{c_{m-1}}{\longrightarrow} o_{m}
$$

The final quality of $o_{m}$ w.r.t. $S S$ is defined by

$$
\operatorname{FinQual}\left(o_{m}, S S\right)=\mathrm{QA}\left(o_{1}\right) \times \prod_{j=1}^{m-1} \mathrm{QF}\left(c_{j}\right) \text {. }
$$

Given any object $o$ to be synthesized, let us define BestQual $(o)=\{S S \mid S S$ is a synthesis sequence for synthesizing object $o$ such that FinQual $(o, S S)=q$ and there is no synthesis sequence $S S^{\prime}$ for $o$ such that Finqual $\left.\left(o, S S^{\prime}\right)<q\right\}$. Thus, BestQual $(o)$ is the set of all synthesis sequences for synthesizing object $o$ that yield the "highest" possible quality.

The first problem that an end-user may wish to pose is the following:

Maximal Quality at Minimal Cost Object Synthesis Problem (BestquallstCost.)

In this problem, the user wishes to first synthesize object $o$ at the maximal quality-level possible and subsequently minimize the total cost. In other words, quality is the primary concern, while cost is to be minimized only after the optimal quality is achieved.

Formally, this problem can be specified as follows: Let BestQualLstCost $(o)=\{S S \mid S S \in$ BestQual $(o)$ and there is no synthesis sequence $S S^{\prime} \in \operatorname{BestQual}(o)$ such that TotCost $\left(\right.$ SS $\left.^{\prime}\right)<$ TotCost(SS)\}. 
For an algorithm to correctly solve this problem, given any object $o$ to be synthesized w.r.t. a COMS $\Gamma$, the algorithm must return a synthesis sequence in BestQual $(o)$. We show below how both the OSA and the OptOSA algorithms may be modified to compute synthesis sequences that yield maximal quality objects at the minimal possible cost.

We first replace the operator $T$ by a new operator, $T Q$. Instead of operating on quadruples as $T$ did, $T Q$ operates on 5-tuples obtained by augmenting the quadruples $T$ worked on by a fifth "quality" argument.

\section{Definition 3.9 (Operator $T Q$ )}

Input: Same as operator T (cf. Definition 3.8).

Output: A member of BestQual(o).

1. $T Q^{0}(X . t y p e)=\left\{(X . t, \operatorname{size}(X . t), 0, X . t, \mathrm{QA}(X . t)) \mid(X . t\right.$, size $\left.(X . t), 0, X . t) \in T^{0}(X . t y p e)\right\}$.

2. $T Q^{i+1}(X . t y p e)=T Q^{i}(X . t y p e) \cup(X . t, S, C, S e q, Q) \mid$ there is a 5-tuple $\left(X . t^{\prime}, S 1, C 1, S e q 1, Q 1\right) \in$ $T Q^{i}(X . t y p e)$ where there exists a $c \in \mathcal{H C}(N)$ such that $c\left(X . t^{\prime}\right)=X . t$ and $C=C 1+$ cost_rate $(c) \times$ $S 1$ and

$S=$ size_ratio $(c) \times S 1$ and $S e q=S e q 1 \stackrel{c}{\longrightarrow} X . t$ and $Q=Q 1 \times Q F(c)\}$.

Note that the operator $T Q$ is exactly like the operator $T$ except that it deals with 5 -tuples instead of quadruples - the fifth component being a quality component.

Algorithm 3 QmaxLcost Algorithm for Computing Maximal Quality, Least Cost Synthesis Sequences We use the same algorithm as the OptOSA algorithm, with the following modifications.

1. In Step 1 of the OptOSA algorithm, we assume that nodes have one extra field, denoted qual.

2. Step 3 of the OptOSA algorithm: replace computation of $T^{0}$ (a.type) by $T Q^{0}$ (a.type).

3. In Step 4 of the OptOSA algorithm, we make one additional Assignment: V.qual $:=-1$. (The reason V.qual is set to minus 1 is that we will eventually minimize the the minus of quality which is the same as maximizing quality.)

4. Furthermore, in Step 4, we assume that $X$ is sorted in ascending order on two keys: the primary key is the qual field, the secondary key is the overallcost field. In particular, note that this means that node $V_{1}$ precedes node $V_{2}$ only if either:

- $V_{1}$.qual $<V 2$.qual or

- $V_{1}$.qual $=V 2$.qual and $V_{1}$.overallcost $\leq V_{2}$.overallcost

5. In Step 6(a)(iv), add an extra assignment statement:

$$
V_{c, o^{\prime}} \text {.qual }=\text { Cur.qual } \times \mathbf{Q F}(c) .
$$

6. In Step 6(a)(iv), when inserting $V_{c, o^{\prime}}$ into the list $X$, ensure that $X$ is kept in sorted order w.r.t. the primary key, qual, and subsequently w.r.t. the secondary key overallcost as outlined in item 4 above.

Theorem 3.3 (Soundness and Completeness of the QmaxLcost Algorithm) Suppose $\Gamma=$ $(G, O b j, l o c, \mathcal{H C}, \mathcal{C} \mathcal{A P})$ is a COMS, $G=(V, E), N$ is a node in $V$, and $o$ is an object we wish to synthesize. Then: 
1. If the QmaxLcost algorithm returns a synthesis sequence, then that synthesis sequence is in BestQual $(o)$, i.e. this is an optimal synthesis sequence for object $o$ within node $N$.

2. If BestQual $(o)=\left\{S S_{1}, \ldots, S S_{n}\right\}, n \geq 1$, then the QmaxLcost algorithm is guaranteed to return some $S S_{i}$.

3. The QmaxLcost algorithm returns with failure iff there is no synthesis sequence for object $o$ in node $N$.

4. The first solution found by the QmaxLcost algorithm is guaranteed to be an optimal one.

One problem with the QmaxLcost algorithm is that it may turn out that the cost of synthesizing a high-quality object may be too much. In such cases, a user may wish to indicate a trade-off between cost and quality. To do so, the user may place weights on cost and quality. For instance, the assignment of weights 5 and 1 to quality and cost, respectively, indicates that the user feels that quality is 5 times more important than cost.

In general, suppose $w_{c}$ and $w_{q}$ are positive integers denoting the weights assigned by a given user to cost and quality, respectively. Then we may define the badness of a synthesis sequence $S S=o_{1} \stackrel{c_{1}}{\rightarrow}$ $o_{2} \stackrel{c_{2}}{\longrightarrow} o_{3} \stackrel{c_{3}}{\longrightarrow} \cdots o_{m-1} \stackrel{c_{m-1}}{\longrightarrow} o_{m}$ of an object $\boldsymbol{o}_{m}$ as follows:

$$
\operatorname{BAD}(S S)=w_{c} \times \operatorname{Tot} \operatorname{Cost}(S S)-w_{q}(\operatorname{Finqual}(S S) .
$$

Intuitively, we would like to minimize badness (i.e. minimize cost, maximize quality).

Minimizing baD-ness Problem. The user may be interested in finding a synthesis sequence $S S$ for object $o$ such that there is no other synthesis sequence $S S^{\prime}$ for $o$ such that $\operatorname{BAD}\left(S S^{\prime}\right)<\operatorname{BAD}(S S)$. This is call the problem of finding the minimally bad synthesis sequence for $o$.

This problem can be easily solved by a small modification of the QmaxLcost algorithm. The node structures and all parts of the algorithm remain unchanged except for one item: Instead of the array $X$ being sorted according to two keys, we keep it sorted in ascending order w.r.t. the value of the expression:

$$
w_{c} \times V \text {.overallcost }-w_{q} \times V \text {.qual.. }
$$

With this minor change, the QmaxLcost algorithm works and correctly computes minimally bad synthesis sequences for any object.

\subsection{Collaboration in Distributed-COMS}

In this section, we show how the framework for synthesizing objects within a single node may be easily extended to synthesize objects across a network. We have already shown how an object $o$ may be synthesized entirely within a given node $N$. Suppose now that node $N$ wishes to synthesize object $o$, but instead of doing so entirely within node $N$, it may access data and/or conversion capabilities located at other nodes. This may be modeled as follows.

Definition 3.10 Suppose $\Gamma=(G, O b j, l o c, \mathcal{H C}, \mathcal{C} \mathcal{A P})$ is a COMS where $G=(V, E)$. A send operation is of the form sends(Sender,Object, Recipient) where (Sender, Recipient) $\in E$ - the statement sends(Sender, Object, Recipient) indicates that Sender is sending the specified object to the recipient. 
Definition 3.11 (Distributed Synthesis Sequence) Suppose $\Gamma=(G, O b j, l o c, \mathcal{H C}, \mathcal{C} \mathcal{A P})$ is a COMS where $G=(V, E)$. An object $o_{m}$ may be synthesized by node $N$ just in case there is a sequence

$\left(N_{1}, c_{1}, o_{1}\right), \ldots,\left(N_{m-1}, C_{m-1}, o_{m-1}\right),\left(N_{m}, \star, o_{m}\right)$ such that:

1. $N_{m}=N$ and

2. $N_{1} \in \operatorname{loc}\left(o_{1}\right)$ and

3. For all $1<i \leq m$ :

(a) $N_{i} \in \operatorname{loc}\left(o_{i}\right)$ or

(b) there exists a $j<i c_{j}$ is a conversion capability in $\mathcal{H C}\left(N_{j}\right)$ such that $c_{j}\left(o_{j}\right)=o_{i}$ and $N_{i}=N_{j}$ or

(c) there exists a $j<i$ such that $\left(N_{j}, N_{i}\right) \in E$ and $c_{i}=\operatorname{sends}\left(N_{j}, o, N_{i}\right)$.

We will call

$$
\left(N_{1}, o_{1}\right) \stackrel{c_{1}}{\longrightarrow}\left(N_{2}, o_{2}\right) \stackrel{c_{2}}{\longrightarrow}\left(N_{3}, o_{3}\right) \stackrel{c_{3}}{\longrightarrow} \cdots\left(N_{m-1}, o_{m-1}\right) \stackrel{c_{m-1}}{\longrightarrow}\left(N_{m}, o_{m}\right)
$$

a distributed synthesis sequence for object $o_{m}$ w.r.t. node $N$.

The main idea behind distributed synthesis sequences is that they allow a node to perform an arbitrary sequence of operations within the node on one or more objects and then send the results to another node that may then do the same. This process can be continued till the desired object is synthesized. The operator $T C$ described below captures the above process.

\section{Definition 3.12 (Operator $T C$ )}

\section{Input:}

- A COMS $\Gamma=(G, O b j, l o c, \mathcal{H C}, \mathcal{C} \mathcal{A P})$ where $G=(V, E)$

- A node $N$ in $V$ and

- An object $o$ of the form $X$.type that we wish to synthesize in an optimal manner.

Output: An optimal synthesis sequence for object $o$.

1. $T C^{0}(X . t y p e)=\{(X . t, \operatorname{size}(X . t), 0,(X . t, N), N) \mid N \in \operatorname{loc}(X . t)\}$.

2. $T C^{i+1}(X . t y p e)=T C^{i}(X . t y p e) \cup\left\{(X . t, S, C, S e q, N 1) \mid\right.$ there is a 5 -tuple $\left(X . t^{\prime}, S 1, C 1, S e q 1, N 2\right) \in$ $T C^{i}(X . t y p e)$ and either

(a) $N 1=N 2$ and there exists a $c \in \mathcal{H C}(N 1)$ such that $c\left(X . t^{\prime}\right)=X . t$ and $C=C 1+$ cost_rate $(c) \times$ $S 1$ and $S=$ size_ratio $(c) \times S 1$ and $S e q=S e q 1 \stackrel{c}{\longrightarrow}\left(X . t, N_{1}\right)$ or

(b) $(N 1, N 2) \in E$ and $X . t=X . t^{\prime}$ and $C=C 1+\wp(N 1, N 2) \times S 1$ and $S e q=S e q 1 \stackrel{\operatorname{sends}\left(N 2, X . t^{\prime}, N 1\right)}{\longrightarrow}$ $\left.\left(X . t, N_{2}\right)\right\}$. 
Intuitively, if the 5-tuple (X.t, size (X.t), S,C,Seq, N1) appears in $T C^{i}$ for some $i$, then this means that node $N 1$ can synthesize object $X$.t of size size $(X . t)$ using the distributed synthesis sequence $S e q$ and incur a cost of at most $C$ in the process of doing so. Note that it is entirely possible that $T C^{i}$ may contain two or more tuples that are identical in all attributes execept for the cost and distributed synthesis sequence attributes - these will correspond to two or more ways in which object $X . t$ can be synthesized at node $N$.

Lemma 3.2 (Properties of $T C$ Operator) Consider a COMS $\Gamma$, a node $N$ in $\Gamma$, and an object $o$ of the form X.type that we wish to synthesize w.r.t. node $N$. For all $j$, if $T C^{j}$ (X.type) contains a 5-tuple of the form $\left(o^{\prime}\right.$, size $\left.\left(o^{\prime}\right), C^{\prime}, S S^{\prime}, N\right)$, then there is a distributed synthesis sequence $S S^{\prime}$ of object $o^{\prime}$ w.r.t. node $N$.

It is easy to see that we can easily modify both the OSA and the OptOSA algorithms to compute optimal distributed synthesis sequences.

\subsubsection{The Distributed OSA Algorithm (DOSA)}

In this section, we present an algorithm that extends the OSA algorithm to handle the construction of synthesis sequences across multiple nodes in a COMS.

\section{Algorithm 4 (Distibuted Object Synthesis Algorithm (DOSA))}

\section{Input:}

- A COMS $\Gamma=(G, O b j, l o c, \mathcal{H C}, \mathcal{C} \mathcal{A} \mathcal{P})$ where $G=(V, E)$

- A node $N$ in $V$ and

- An object $o$ to be synthesized in node $N$.

Tried: A global set variable initialized to $\emptyset$.

1. Let Name(o) be of the form str.type.

2. Let $X_{1}=\left\{\left(c\right.\right.$, Ob.type $\left.1, N_{1}\right) \mid c$ is a conversion capability in $\mathcal{H C}\left(N_{1}\right)$ and $N_{1}=N$ Name $(c($ Ob.type 1$))=$ str.type $\}$. (* Note that $O b$ may not necessarily be such that $N_{1} \in \operatorname{loc}(O b)^{*}$ ).

3. Let $X_{2}=\left\{\left(\operatorname{sends}\left(N_{1}\right.\right.\right.$, Ob.type $\left.1, N_{2}\right)$,Ob.type1, $\left.N_{1}\right) \mid(N 1, N 2) \in E$ and $N$ ame $($ Ob.type 1$)=$ str.type $\}$ and $N_{2}=N$.

4. Let $X=X_{1} \bigcup X_{2}$.

5. if $\{x \mid x \in X \& x \notin$ Tried $\}=\emptyset$ then return failure and halt.

6. Otherwise (* i.e. $X$ still has something in it that can be processed *)

7. if there exists a $\left(c, O b, N_{1}\right) \in X$ such that $\left(c, O b, N_{1}\right) \notin$ Tried and such that $N_{1} \in \operatorname{loc}(O b)$ then return

$$
S O L=\left(O b, N_{1}\right) \stackrel{e}{\longrightarrow}\left(\text { str.type }, N_{1}\right)
$$

and halt.

8. Otherwise if there exists a $\left(\operatorname{sends}\left(N_{1}, O b, N_{2}\right), O b, N_{1}\right) \in X$ such that $\left(\operatorname{sends}\left(N_{1}, O b, N_{2}\right), O b, N_{1}\right) \notin$ Tried and such that $N_{1} \in \operatorname{loc}(O b)$ then return

$$
S O L=\left(O b, N_{1}\right) \stackrel{\operatorname{sends}\left(N_{1}, O b, N_{2}\right)}{\longrightarrow}\left(\text { str.type, } N_{2}\right),
$$

and halt. 
9. Otherwise, non-deterministically select a pair (oper, $\left.O b, N_{1}\right) \in X$.

10. Set $X$ to $X-\left\{\left(\right.\right.$ oper, $\left.\left.O b, N_{1}\right)\right\}$ and set Tried to Tried $\cup\left\{\left(\right.\right.$ oper, $\left.\left.O b, N_{1}\right)\right\}$.

11. If the call $\operatorname{OSA}\left(\Gamma, N_{1}, O b\right)$ succeeds and returns $S O L 1$, then

(a) if oper $=c$ then

$$
S O L=S O L 1 \stackrel{c}{\longrightarrow}\left(\text { str.type }, N_{1}\right) .
$$

Return SOL. Halt with success.

(b) if oper $=\operatorname{sends}\left(N_{1}, O b, N_{2}\right)$ then

$$
S O L=S O L 1 \stackrel{\operatorname{sends}\left(N_{1}, O b, N_{2}\right)}{\longrightarrow}\left(\text { str.type, } N_{2}\right) .
$$

Return SOL. Halt with success.

12. Otherwise, goto Step 5.

\subsubsection{The Distributed OptOSA Algorithm (DOptOSA)}

In this section, we present an algorithm that extends the DOptOSA algorithm to handle the construction of optimal synthesis sequences across multiple nodes in a COMS.

\section{Algorithm 5 (Distributed Optimal Object Synthesis Algorithm (DOptOSA))}

\section{Input:}

- A COMS $\Gamma=(G, O b j, l o c, \mathcal{H C}, \mathcal{C} \mathcal{A P})$ where $G=(V, E)$

- A node $N$ in $V$ and

- An object $o=$ a.type to be synthesized in an optimal manner.

Output: An optimal synthesis sequence for object $o$ in node $N$.

1. Our algorithm uses a special node structure that has the following fields: name, size, cost, seq, overallcost, loc, next.

2. SOL $:=N I L$

3. Compute $T C^{0}$ (a.type).

4. For each five-tuple of the form (a.t, size $(a . t), 0$, a.t, $\left.N_{1}\right)$ in $T^{0}$ (a.type), create a node $V$ having

$$
\begin{gathered}
V \cdot \text { name }=a . t ; V \cdot \operatorname{size}=\operatorname{size}(a \cdot t) ; \\
V \cdot \operatorname{cost}=0 ; V \cdot \text { seq }=a . t ; V \cdot \text { overallcost }=0 ; V \cdot \operatorname{loc}=N_{1} .
\end{gathered}
$$

Let $X$ be a linked list consisting of all these nodes.

(* We will always assume that $X$ is sorted in ascending order according to the overallcost field. As initially all these nodes have their overallcost field set to 0 , this means that when this step is first executed, any ordering will suffice. ${ }^{*}$ )

5. if $X=N U L L$ then halt with failure.

6. else 
(a) i. Cur $:=\operatorname{head}(X) ; X:=\operatorname{tail}(X)$;

ii. if $C$ ur.name $=$ a.type then return $C$ ur.seq and halt.

iii. Cand $_{1}:=\left\{\left(c, o^{\prime}, N\right) \mid c \in \mathcal{H C}(N)\right.$ and $c($ Cur.name $)=o^{\prime}$ and $N \in \operatorname{loc}\left(o^{\prime}\right)$ and $N=$ Cur.loc $\}$;

iv. For each $\left(c, o^{\prime}, N_{1}\right) \in C$ and create a node $V_{c, o^{\prime}, N_{1}}$ with fields:

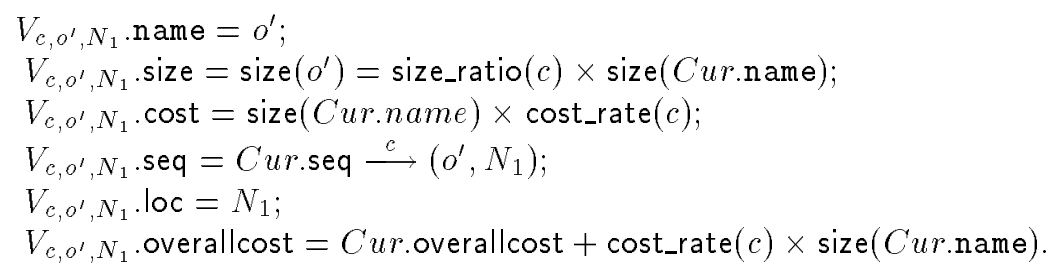

if there is not a node $M$ in $V_{c, o^{\prime}, N_{1}}$.seq such that $V_{c, o^{\prime}, N_{1}}$.name $=M$.name and $V_{c, o^{\prime}, N_{1}}$.size $\geq$ $M$ size then insert $V_{c, o^{\prime}, N_{1}}$ into the list $X$, keeping $X$ in sorted order w.r.t. the tot cost field.

v. $\operatorname{Cand}_{2}:=\left\{\left(\operatorname{sends}\left(N_{1}, o^{\prime}, N_{2}\right), o^{\prime}, N_{1}\right) \mid C u r\right.$.name $=o^{\prime}$ and $(N 1, N 2) \in E$ and $N_{1} \in \operatorname{loc}\left(o^{\prime}\right)$ and $N_{1}=$ Cur.loc $\}$;

vi. For each $\left(\operatorname{sends}\left(N_{1}, o^{\prime}, N_{2}\right), o^{\prime}, N_{1}\right) \in \operatorname{Cand}_{2}$ create a node $V_{o^{\prime}, N_{2}}$ with fields:

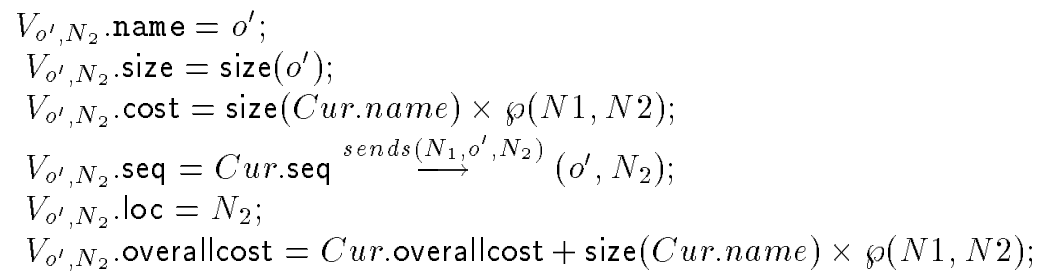

if there is not a node $M$ in $V_{o^{\prime}, N_{2}}$.seq such that $V_{o^{\prime}, N_{2}}$.name $=M$.name and $V_{o^{\prime}, N_{2}}$.size $\geq$ $M . s i z e$ then insert $V_{o^{\prime}, N_{2}}$ into the list $X$, keeping $X$ in sorted order w.r.t. the totcost field.

vii. Goto Step 5.

\section{Implementation and Experiments}

We have implemented the OSA, OptOSA, DOSA and DOptOSA algorithms on a SUN workstation running Unix. The OSA and OptOSA algorithms included about 1300 lines of C-code. The DOSA and DOptOSA algorithms comprise about 1300 lines of $\mathrm{C}$ code as well. We will now describe the experiments we conducted. Each point shown in the graphs reflecting experimental results was obtained by averaging the results of various runs.

\subsection{OSA vs. OptOSA: Cost of Synthesis Sequence}

We ran experiments to compare the cost of a synthesis sequence computed by the OSA algorithm against the cost of a synthesis sequence computed by the OptOSA algorithm. In this experiment, we varied the number of objects at a given node from 50 to 600 at intervals of 50 . The number of types of these objects were varied from between 5 and 30 at steps of 10 .

Figure 3 shows the graph describing the costs of synthesis sequences computed by OSA as opposed to the costs of synthesis sequences computed by OptOSA. As can be easily seen, OptOSA performs significantly better than OSA - it consistently yields better results than OSA. Both OSA and OptOSA produce better and better synthesis sequences as the number of objects is increased. Both algorithms 


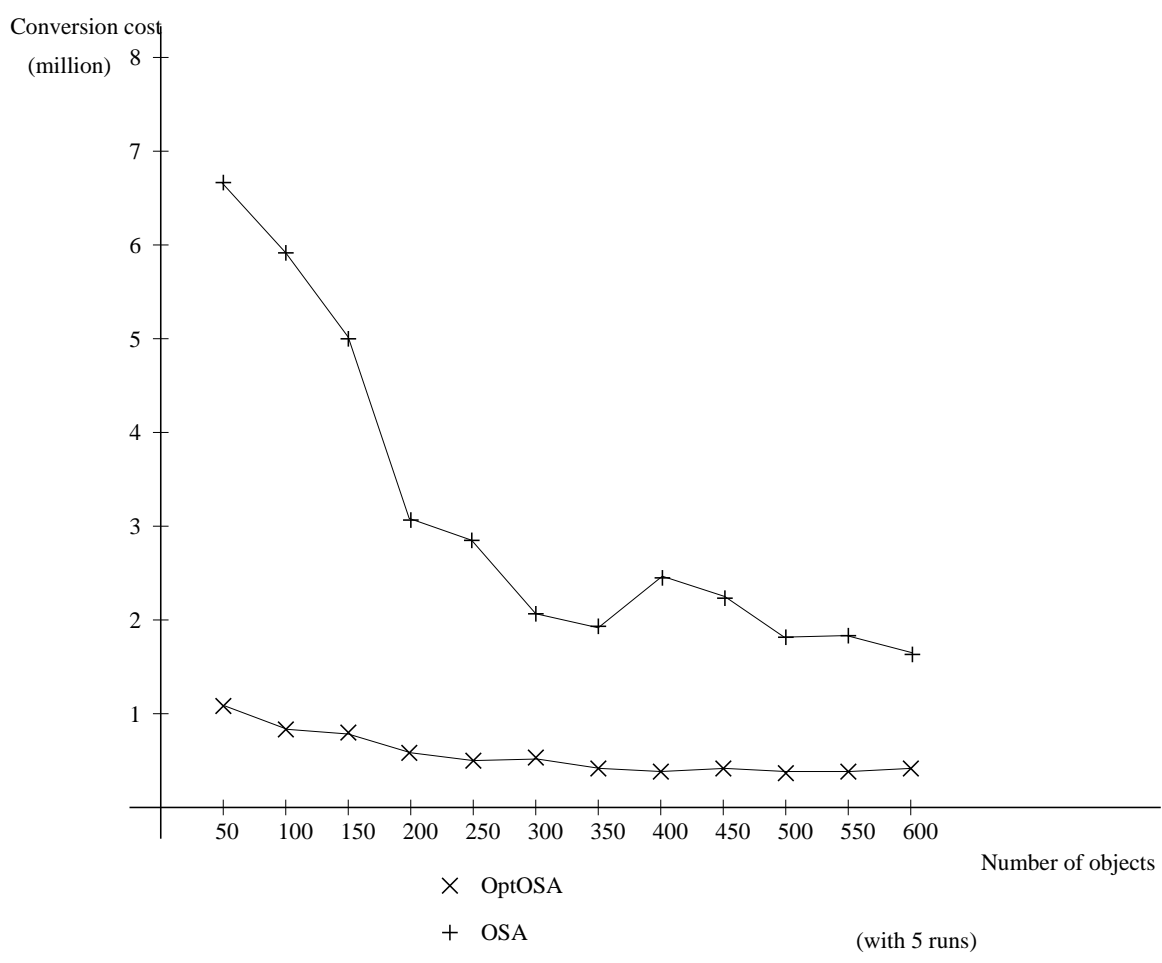

Figure 3: OSA vs. OptOSA: Cost of Synthesis Sequences Generated

appear to reach a "steady state" after 250 objects (in the case of OptOSA) and 500 in the case of OSA. In this steady state, OptOSA computes synthesis sequences that are about $40 \%$ as expensive as those computed by OSA. Before the steady state, OptOSA computes synthesis sequences that are well below $40 \%$ as expensive as those computed by OSA.

\subsection{OSA vs. OptOSA: Running Times}

The "cheaper" synthesis sequences computed by OptOSA have an attached price tag - OptOSA takes longer to compute these sequences. As Figure 4 shows, OSA takes significantly less time to compute synthesis sequences than does OptOSA. In fact, OSA exhibits remarkably "constant" behavior in terms of running time - it is largely independent of the number of objects being dealt with and seems to take about 1 millisecond for all the cases we tried. In contrast, OptOSA's computation time increases as more objects are present. Furthermore, OSA may compute a synthesis sequence in as much as $\frac{1}{10}$ 'th to $\frac{1}{20}$ "th the time taken by OptOSA. However, in terms of "absolute times", this is not very much and only involves a few milliseconds of savings. In contrast, the synthesis sequence computed by OSA may be inferior to the one computed by OSA in terms of cost.

\subsection{OSA vs. OptOSA: Impact of Conversion Ratio}

In the experiments reported thus far, we have reported on the running time taken by and the cost of the synthesis sequences computed by the OSA and OptOSA algorithms. However, these factors do not take into account, the number of conversion functions. Recall that each object has a name of the form name.type. In our experiment, we allowed the number of types considered to vary from 5 to 30 (in steps of 5) and the number of names to also vary from 5 to 30 (also in steps of 5 ). The total number of 


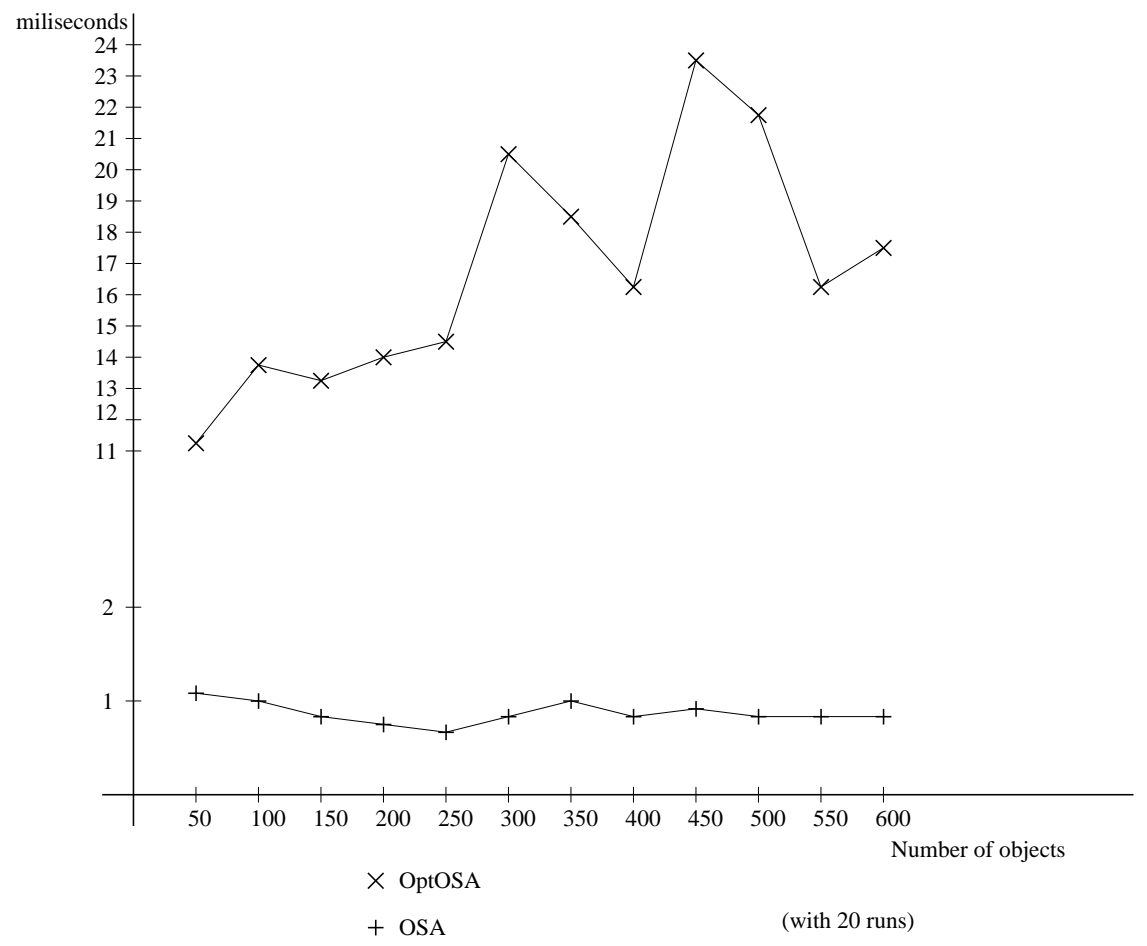

Figure 4: OSA vs. OptOSA: Running Times

conversion functions varied from 5 to 275 in steps of 30 . For any given number of types num $m_{t}$, number of names num $_{n}$, and number of conversion functions num $m_{c f}$, the conversion ratio is defined to be

$$
\text { Conversion Ratio }=\frac{n u m_{t} \times n u m_{n}}{n u m_{c f}} .
$$

In this experiment, we varied the conversion ratio from 2 to 125 - the higher the conversion ratio, the larger the set of potential objects to the set of actual conversion functions available.

\subsubsection{Impact of Conversion Ratio on Cost of Synthesis Sequence Found}

Figure 5 shows the cost of a synthesis sequence found by the OSA algorithm, while Figure 8 shows the cost of a synthesis sequence found by the OptOSA algorithm.

As can be seen from the Figures, as the conversion ratios increase, both algorithms exhibit similar behavior, and the number of objects participating seems to have less of an impact. In the long run, the OptOSA algorithm seems to find solutions that are only half as expensive as the OSA algorithm.

\subsubsection{Impact of Conversion Ratio on Running Time}

Figure 7 shows the time taken by the OSA algorithm to compute a synthesis sequence, while Figure 8 shows the time taken by the OptOSA algorithm.

It is easy to see from the above figures that both of the algorithms exhibit some behavioral peaks when the conversion ratio is 4 . What is important is that as the conversion ratios get larger, the effect of the number of objects decreases, and each algorithm seems to "settle" down to a steady state. In the case of the OSA algorithm, this means that when the conversion ratio is sufficiently high (over 15 


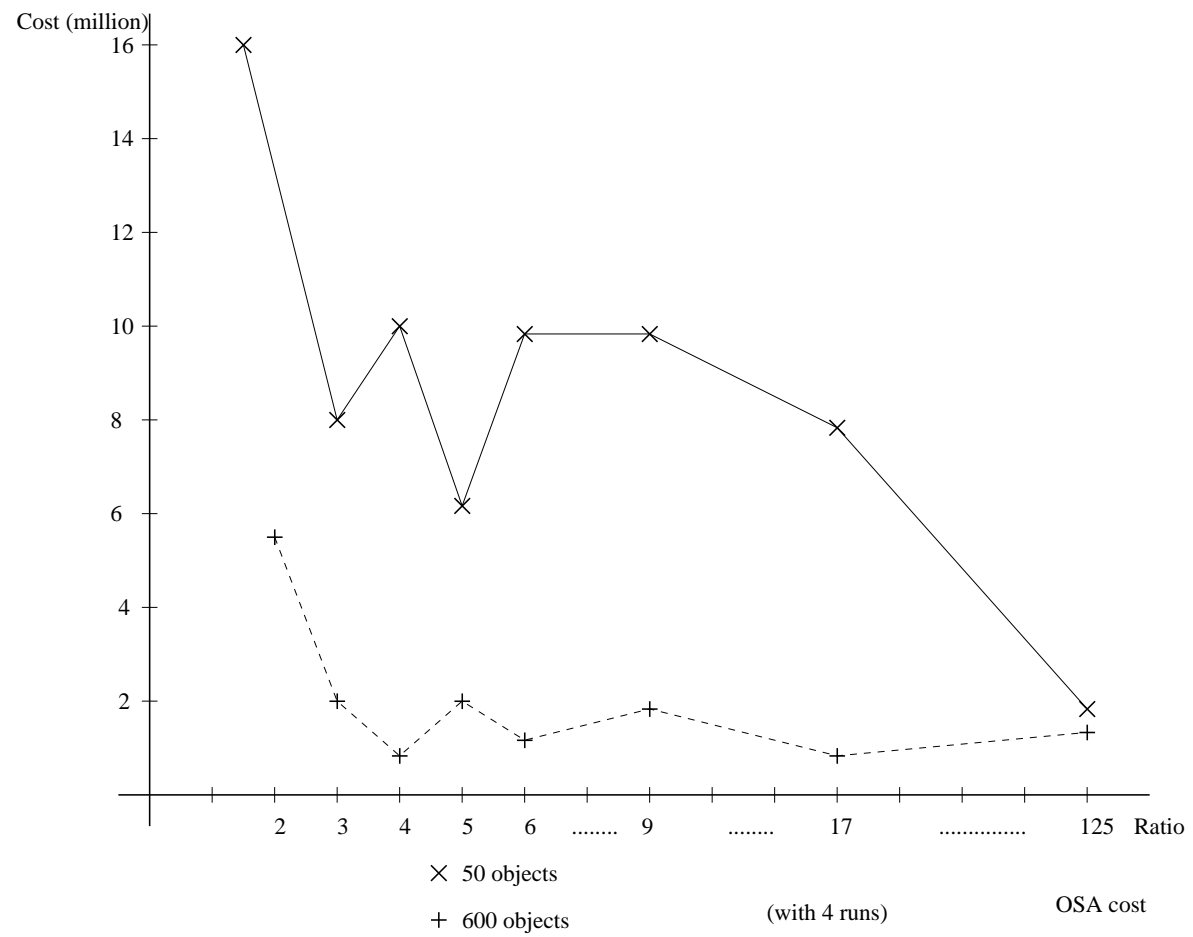

Figure 5: Cost of Solution found by OSA algorithm with Varying Conversion Ratios

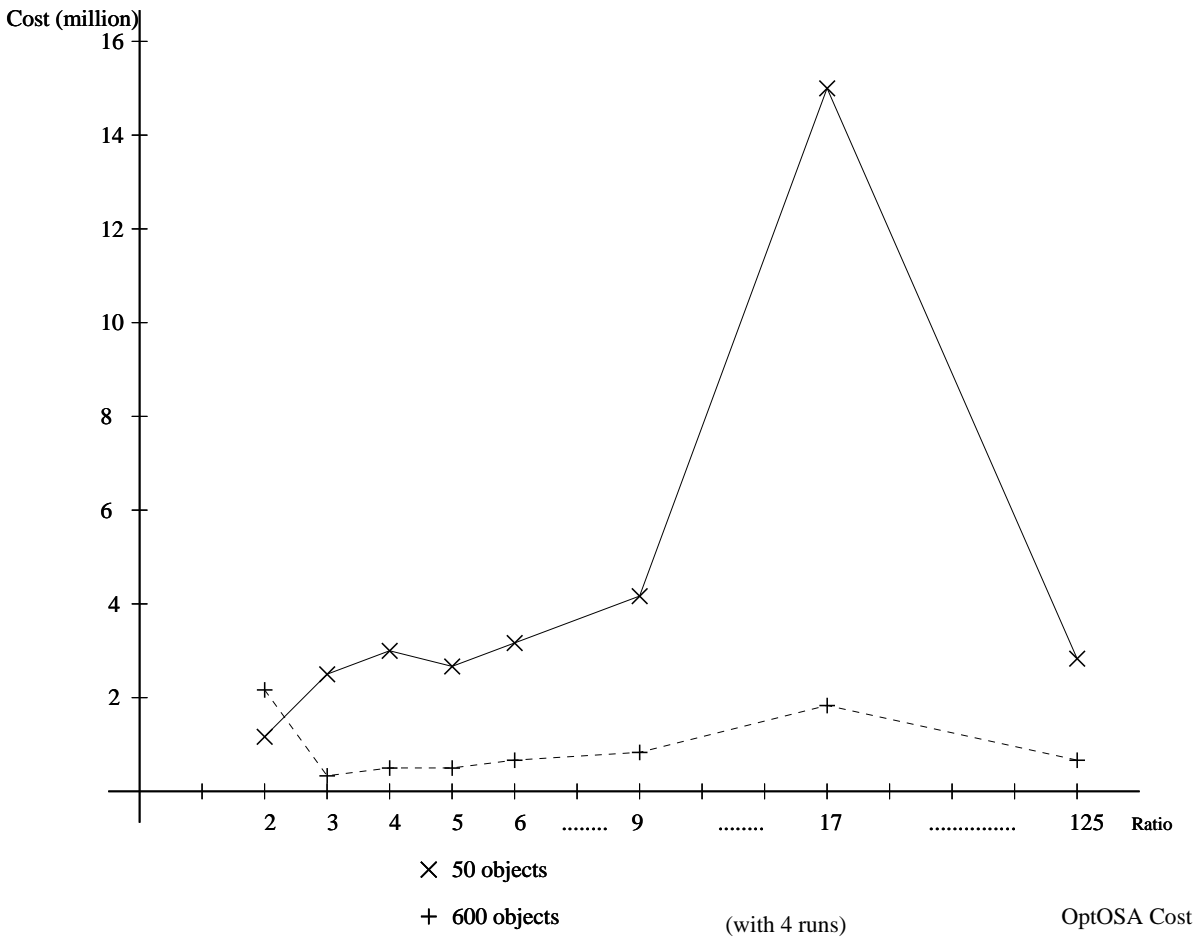

Figure 6: Cost of Solution found by OptOSA algorithm with Varying Conversion Ratios 


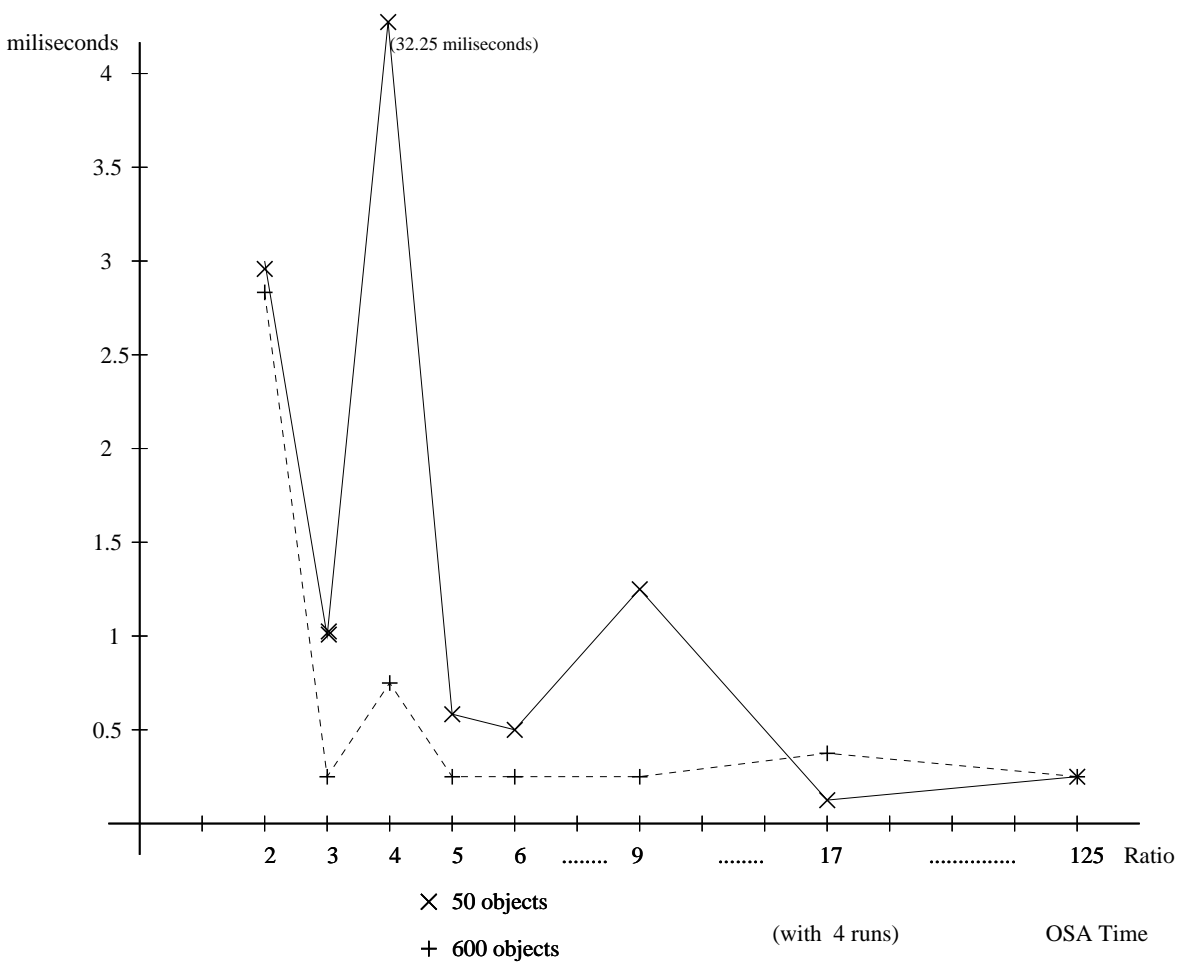

Figure 7: Running Time of OSA algorithm with Varying Conversion Ratios

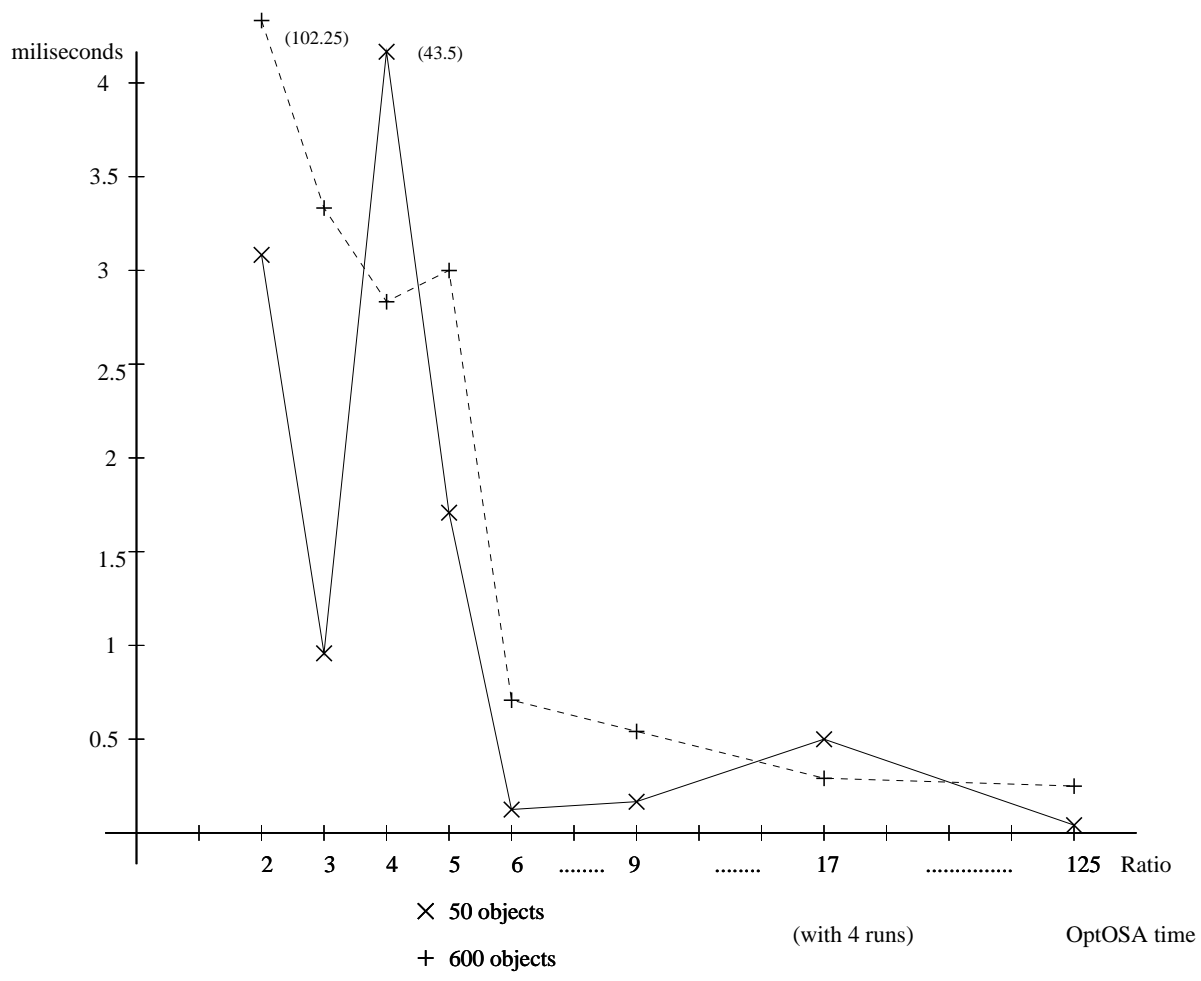

Figure 8: Running Time of OptOSA algorithm with Varying Conversion Ratios 


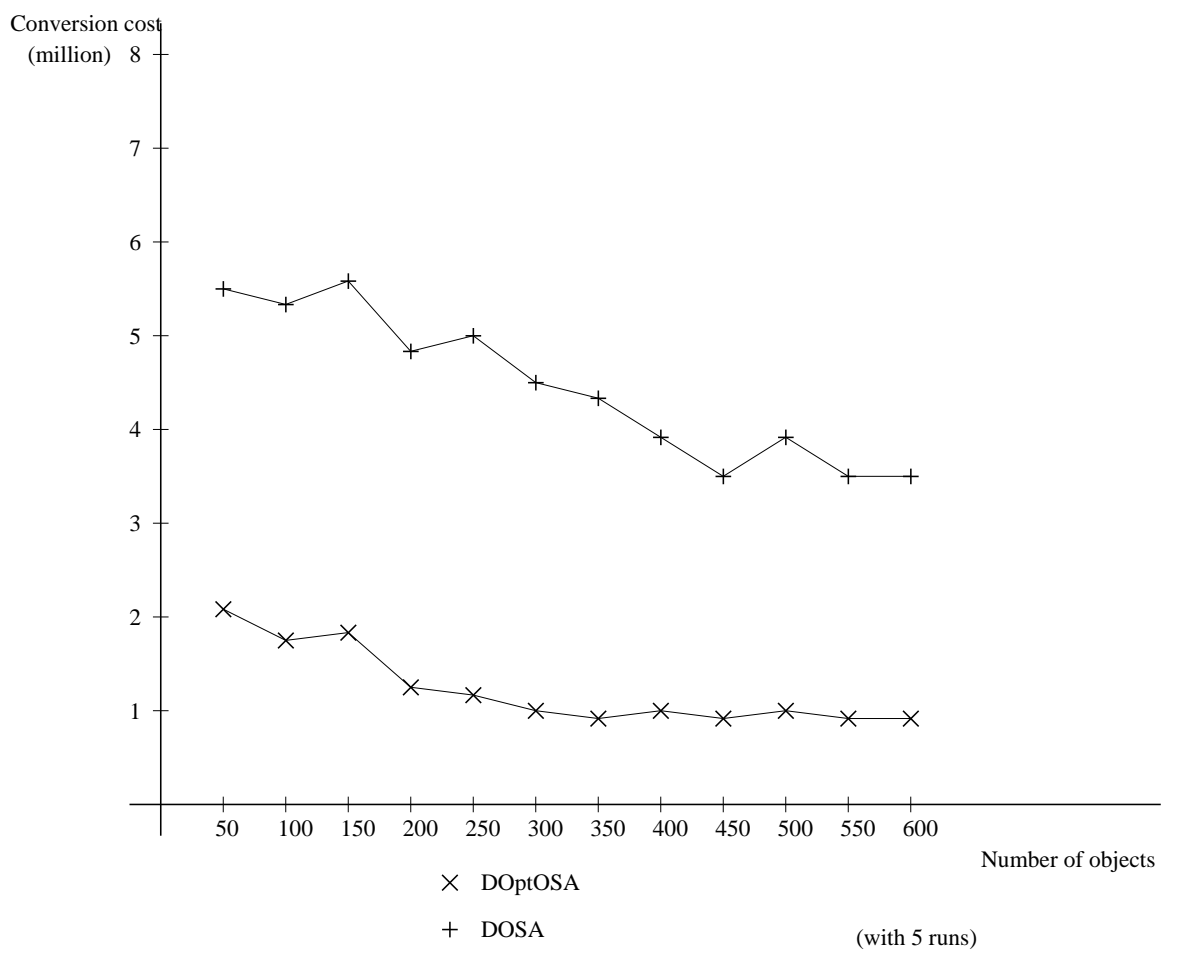

Figure 9: DOSA vs. DOptOSA: Cost of Synthesis Sequences Generated

or so), the OSA algorithm seems to find solutions in about 0.25 seconds. In the case of the OptOSA algorithm, again when the conversion ratio is 15 or so, the OptOSA algorithm seems to find solutions in about 2 seconds. This means that in the long run, we seem to be able to say that the OptOSA algorithm will take about 4 times the time taken by the OSA algorithm, but will find a solution that is half as expensive.

\subsection{DOSA vs. DOptOSA: Cost of Synthesis Sequence}

Figure 9 shows the cost of synthesis sequences computed by the DOSA and the DOptOSA algorithms, respectively, as the number of objects is increased from 50 to 600 . The graph indicates that DOSA and DOptOSA yield solutions that become progressively "less" expensive as the number of objects increases; however, DOptOSA yields synthesis sequences that are only about $\frac{1}{4}$ 'the the cost of the synthesis sequences yielded by DOSA.

\subsection{DOSA vs. DOptOSA: Running Times}

Figure 10 shows the time taken by DOSA and DOptOSA to compute synthesis sequences as the number of objects increase. As seen, DOSA performs about 30-50 times as fast as DOptOSA; however, once again, as in the case of OSA vs. OptOSA, this difference is still measured in a few milliseconds (12-24 milliseconds). What is more interesting, however, is the fact that the time taken by DOSA to compute synthesis sequences decreases as the number of objects increases, while the corresponding time taken by DOptOSA increases. This suggests that in applications where a very large number of objects (in the thousands) are being worked on collaboratively, it might be wiser to use DOSA rather than DOptOSA. 


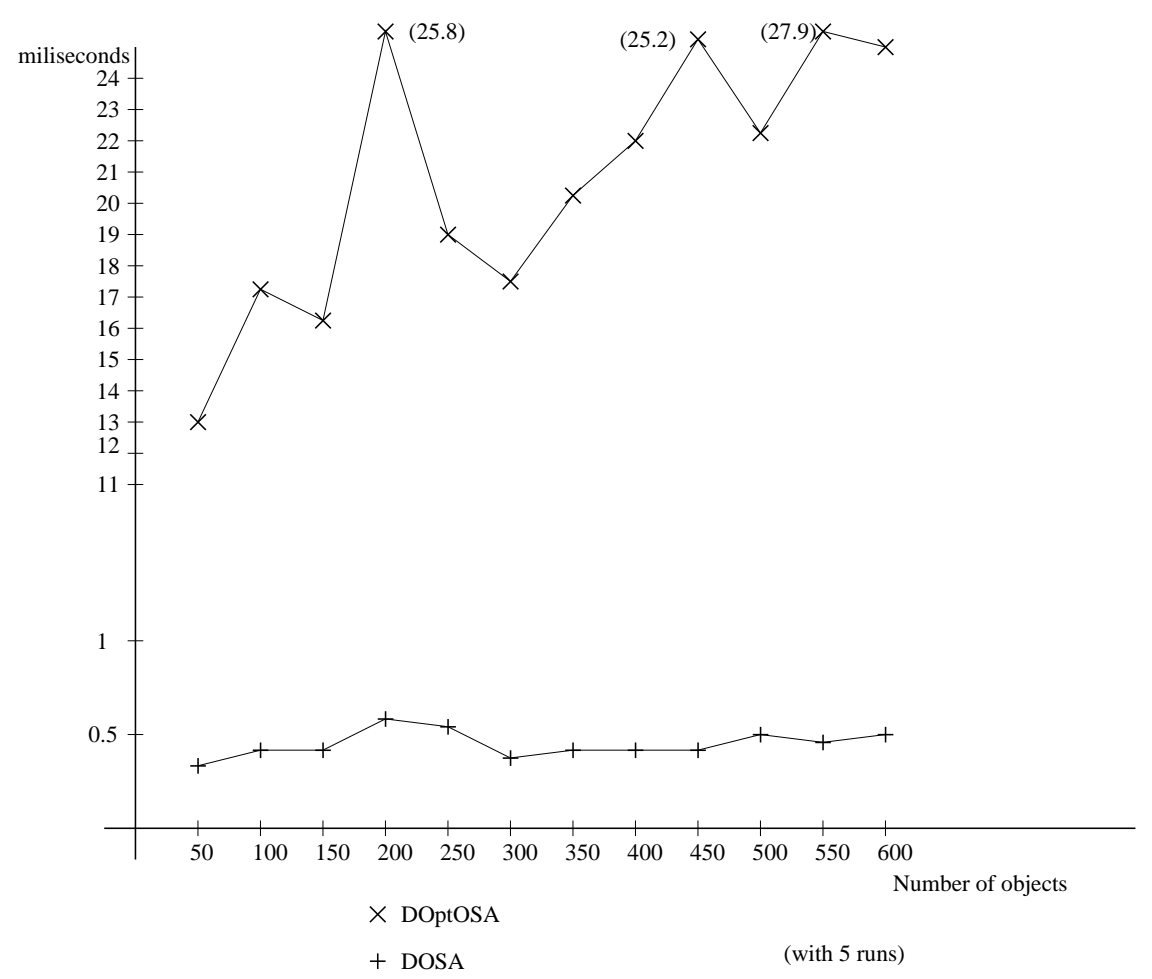

Figure 10: DOSA vs. DOptOSA: Running Times

\subsection{DOSA vs. DOptOSA: Impact of Conversion Ratio}

We also conducted experiments to determine the impact of conversion ratio in the case of DOSA vs. DOptOSA.

\subsubsection{Impact of Conversion Ratio on Cost of Synthesis Sequence}

When the conversion ratio was varied from 2 to 125, we observed (cf. Figure 11) that DOSA returned quickly converged to a stable state when the ratio is around 17 . Furthermore, the number of objects involved did not have a significant impact on the cost of the computed solution. In contrast, DOptOSA (cf. Figure 12) seemed to compute solutions of more or less the same cost as DOSA when the conversion ratio was 17 or higher. However, when the conversion ratio is small, DOptOSA yields solutions that are significantly cheaper.

\subsubsection{Impact of Conversion Ratio on Running Time}

Finally, in terms of running time, DOSA (cf. Figure 13) quickly reached a steady state when the conversion ratio was around 17. However, DOptOSA was less predictable (cf. Figure 14). DOptOSA took about 5-50 times as long as DOSA to find a solution. Again, in absolute terms, this does not seem to make a big impact, merely adding as much as 10-20 milliseconds to the time required by DOSA to find a solution. 


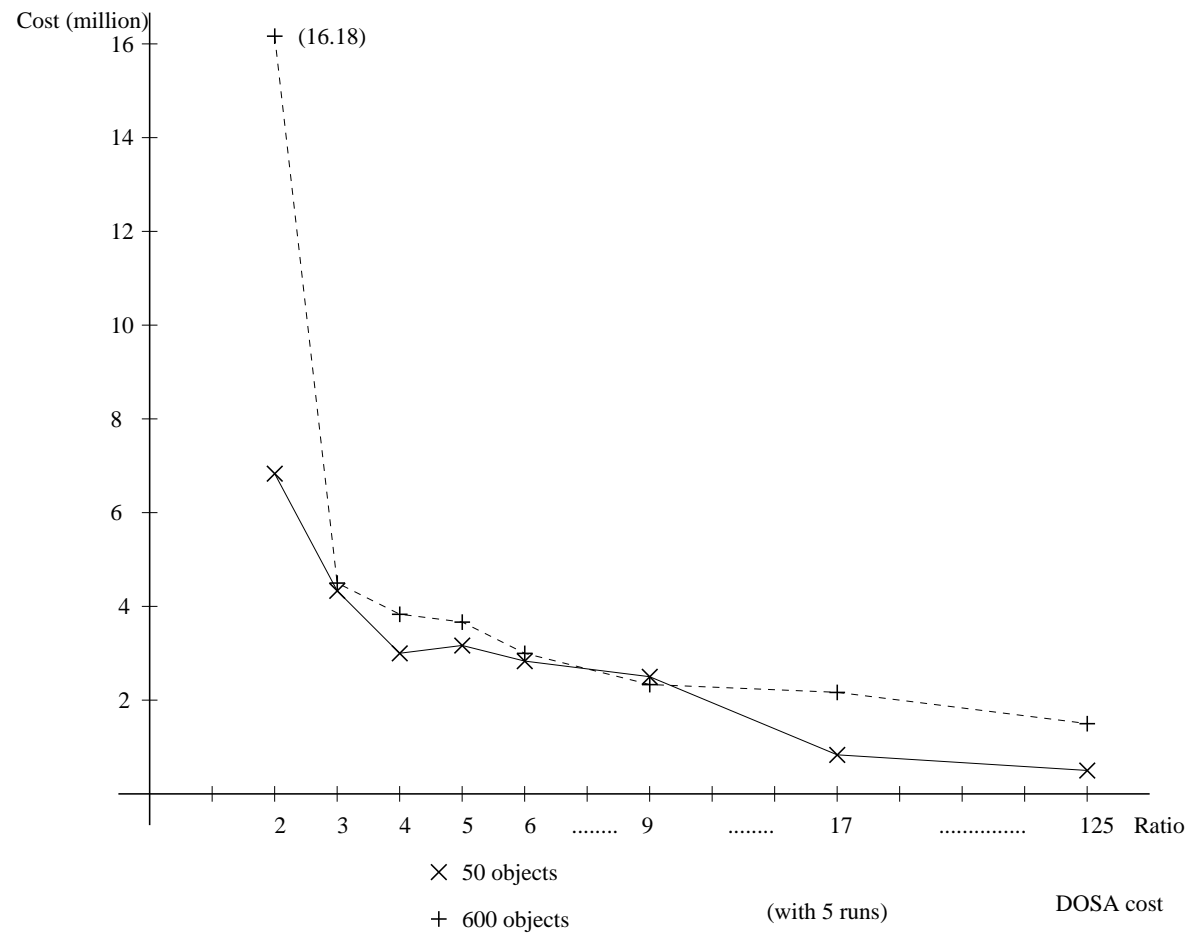

Figure 11: Cost of Solution found by DOSA algorithm with Varying Conversion Ratios

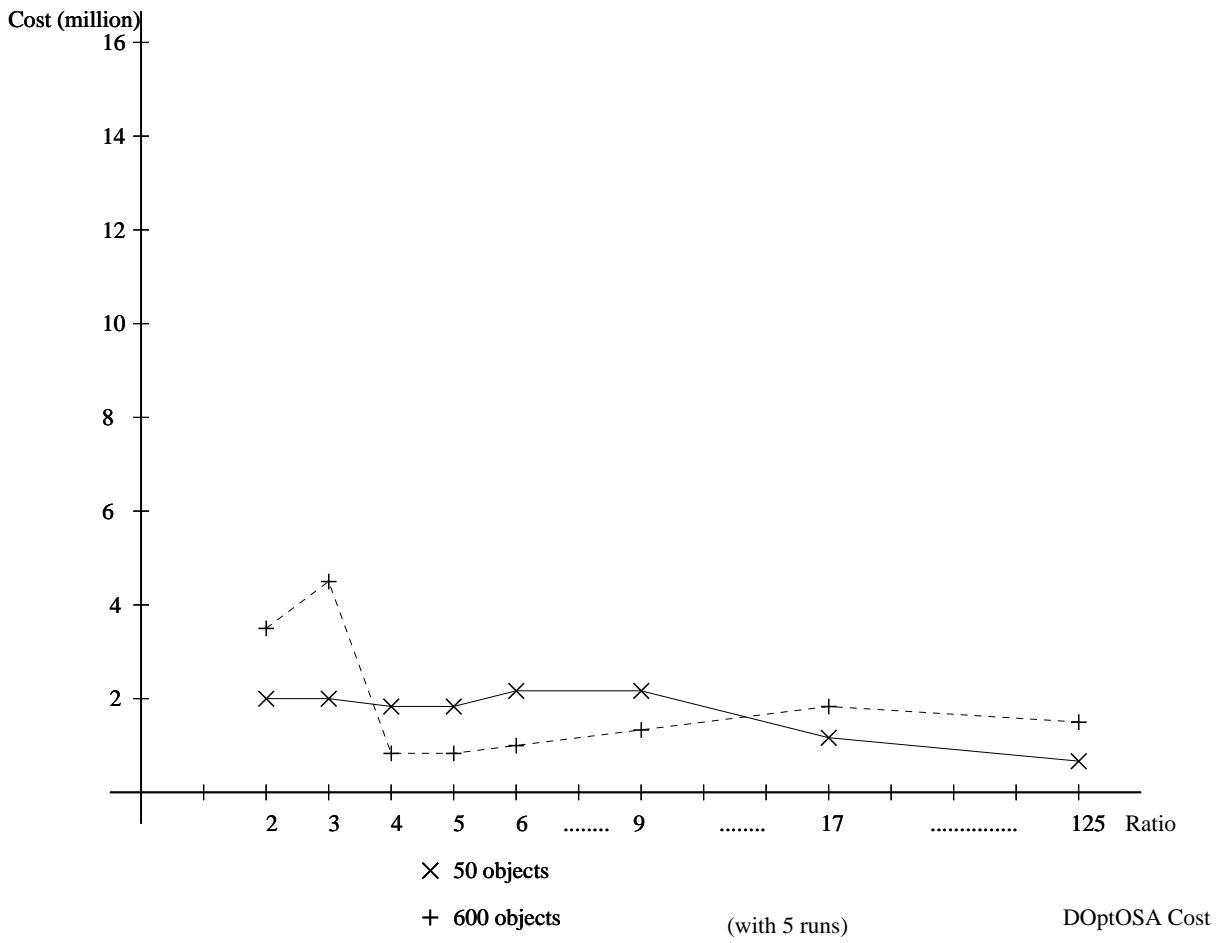

Figure 12: Cost of Solution found by DOptOSA algorithm with Varying Conversion Ratios 


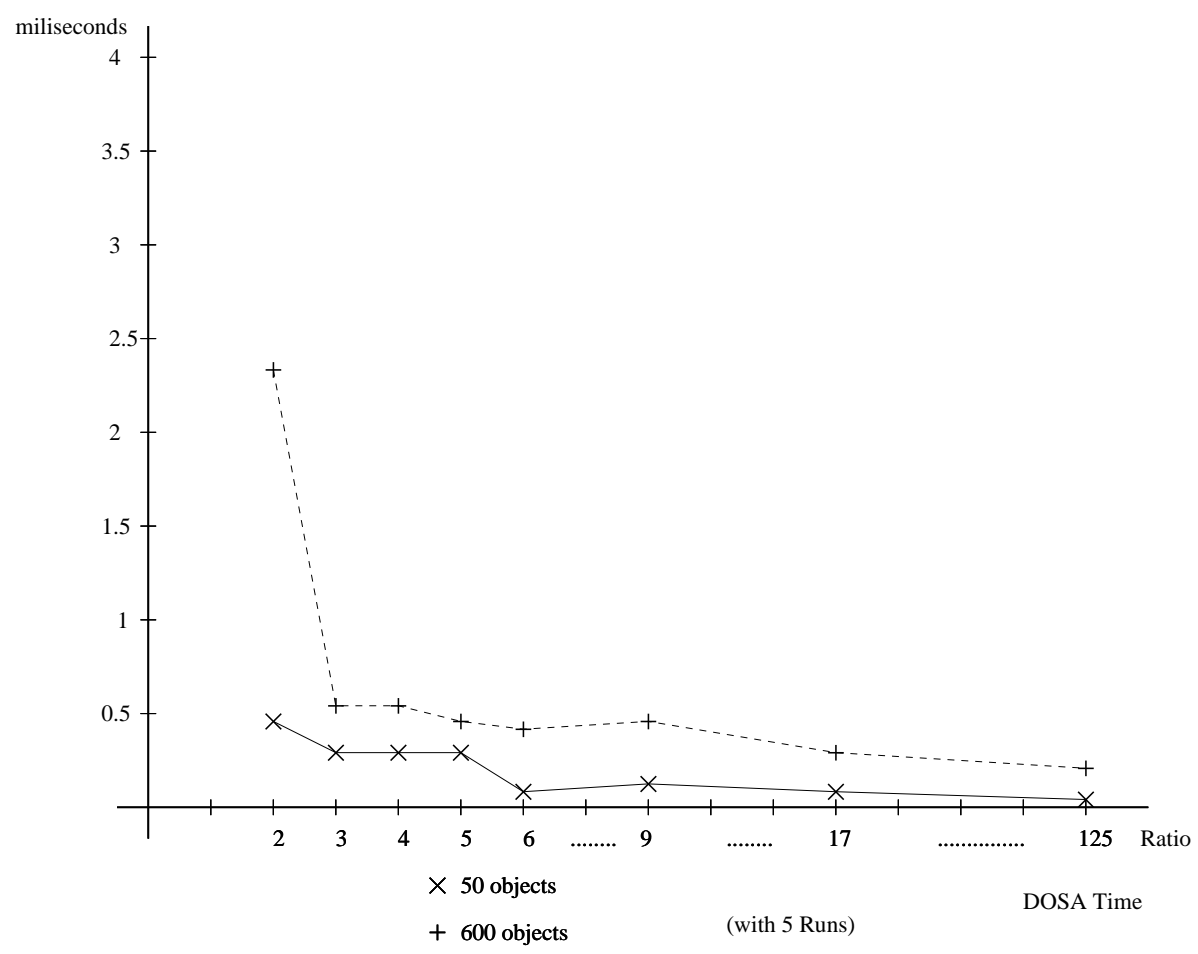

Figure 13: Running Time of DOSA algorithm with Varying Conversion Ratios

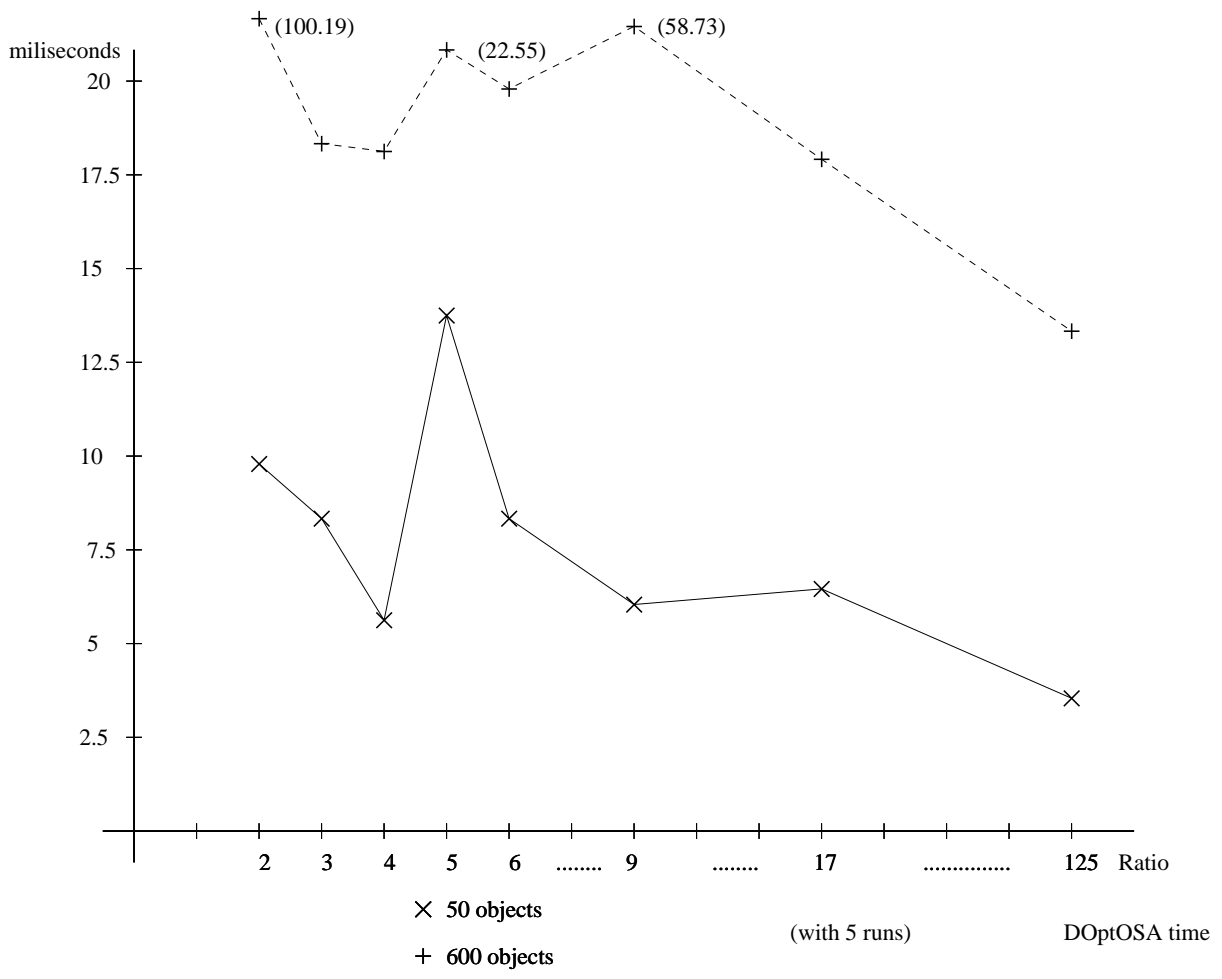

Figure 14: Running Time of DOptOSA algorithm with Varying Conversion Ratios 


\section{$5 \quad$ Related Work}

Multimedia collaboration has been a topic of wide interest. In addition to significant amount of work in the areas of $\mathrm{CSCW}$, recent work in this area includes the hypermedia conversation recording system of Imai et al.[2], multipoint conferencing system of Gong [3], and Argo [4]. In [2], Imai et al. show how to record the artifacts of a realtime collaboration so that when the collaboration is concluded, the collaborators have access not only to the final document, but also to the artifacts (handwritten notes, voice annotations etc.) that led them to this document. Gong [3] studies some of the important issues in multimedia conferencing over packet switched networks, and provides solutions to the problems that arise in multipoint audio and video control. The Argo system [4] on the other hand, is built to let users collaborate remotely using video, audio, shared applications, and whiteboards. Synchronization has been studied by Manohar [5]. They study methods to enable the faithful replay of multimedia objects under varying system parameters. To accomplish synchronization of different session objects, they provide an adaptive scheduling algorithm. Wolf et al [6] show how an application can be shared among heterogeneous systems. They compare two methods for heterogeneous sharing: one optimizes transmission in the system and other optimizes conversions between objects. Ahuja's group at AT\&T [7] also has had significant contributions in collaborative services. They propose a method for generating visual representations of recorded histories of distributed collaborations, so that remote collaborators can easily access information that will let them understand how the collaborative environement evolved to a particular state. Little [8] has presented an elegant document management system for shared data and provided a data model ( $P O M$ ) which permits dynamic compositions of mixed-media documents. Wray et al. [9] have built an experimental collaborative environment called Medusa which integrates data from heterogeneous hardware devices. Medusa provides an environment which facilitates rapid prototyping of new applications. Rajan, Vin et al. [10] started some work on formalizing the notion of multimedia collaboration. They provide a basis which can support a wide spectrum of structured multimedia collaborations. Their formalization captures the requirements of various types of interactive and non-interactive collaborations. They also implemented a prototype collaboration management system based on their formalism. However, the papers listed above do not address the complementary problem studied by this paper, viz. arranging for an object to be transmitted (at minimal cost and with the desired quality) to a participant in a collaboration in a form that he can work with.

\section{Conclusions}

In this paper, we have classified media objects into four broad categories: static, quasi-static, temporal, and quasi-temporal, and developed a theory of media objects in which each media object is represented as a 5-tuple. We have then developed a formal definition of a collaborative multimedia system, consisting of collaborators and distributed media objects. We have presented optimal algorithms for collaborative object synthesis: i.e., for constructing multimedia documents by composing together a given set of media objects. These algorithms are then extended to incorporate quality constraints (such as image size) as well as distribution across multiple nodes. We have proved that these algorithms are sound, complete, and optimal (in the case of OptOSA and DOptOSA.) We have implemented these algorithms, and evaluated their performance.

In future work, we will study the problem of collaborative media systems where multiple collaborators are working together, in a collaborative group-session. In such cases, the sharing of these objects must be done in real-time, and editing changes made by one collaborator must be reflected, 
in a synchronized fashion, and in real-time, on the screens/output devices of others. Most current systems that implement such schemes (e.g. the Sun ShowMe repertoire of products) require that all nodes in the collaborative enterprise have certain common products available on them, (viz. the Sun ShowMe system). In Part II of this series of papers [1], we will show how we may build upon the framework presented in this paper to solve this important problem.

\section{References}

[1] K.S. Candan, B. Prabhakaran, and V.S. Subrahmanian. (1995) Collaborative Multimedia Systems: Synchronized Document Authoring, draft manuscript.

[2] T. Imai, K. Yamaguchi, T. Muranaga, "Hypermedia Conversation Recording to Preserve Informal Artifacts in Realtime", ACM Multimedia 94, Pages 417-424

[3] F. Gong, "Multipoint Audio and Video Control for Packet-Based Multimedia Conferencing", ACM Multimedia 94, Pages 425-432

[4] H. Gajewska, "Argo: A System for Distributed Collaboration", ACM Multimedia 94, Pages $433-440$

[5] N.R. Manohar and A. Prakash, "Dealing with synchronization and timing variability in the playback of interactive session recordings", ACM Multimedia 95, Pages 45-56

[6] K.H. Wolf, K. Froitzheim and P. Schulthess, "Multimedia Application Sharing in a Heterogeneous Environment", ACM Multimedia 95, Pages 57-64

[7] A. Ginsberg and S. Ahuja, "Automating envisionment of virtual meeting room histories", ACM Multimedia 95, Pages 65-76

[8] T.M. Wittenburg and T.D.C. Little, “An Adaptive Document Management System for Shared Multimedia Data", IEEE Intl. Conf. on Multimedia Computing and Systems, 1994, Pages 245254.

[9] S. Wray, T. Glauert, and A. Hopper, "The Medusa Applications Environment", IEEE Intl. Conf. on Multimedia Computing and Systems, 1994, Pages 265-274.

[10] S. Rajan, P.V. Rangan, and H.M. Vin, "A Formal Basis for Structured Multimedia Collaborations", IEEE Intl. Conf. on Multimedia Computing and Systems, 1995. 


\section{Appendix: Proofs of Theorems}

Note to Referees: This section can be removed, for space reasons, when the paper is published. It is included here so that the referees can verify the claims made in this paper.

Proof of Theorem 3.1. (1) Let $S S$ be a synthesis sequence for object $o$ within node $N$, and let $S S$ be of the following form:

$$
o_{1} \stackrel{c_{1}}{\longrightarrow} o_{2} \stackrel{c_{2}}{\longrightarrow} \ldots \stackrel{c_{n-1}}{\longrightarrow} o_{n} \stackrel{c_{n}}{\longrightarrow} o
$$

Since $S S$ is a synthesis sequence, and since $o_{1}, c_{1}, \ldots, c_{n}$ are initially in node $N,\left(c_{n}, o_{n}\right)$ is in $X$ after the step 2 of the initial call.

If $n=1$, then the case is trivial. If $O S A$ chooses $\left(c_{n}, o_{n}\right)$ in step 6 , then $S O L=S S=o_{1} \stackrel{c_{1}}{\rightarrow} o$.

Let us assume that the hypothesis is true for all synthesis sequences of length $\leq n$, and let $S S$ be of length $n+1$, i.e. $S S$ is of the following form:

$$
o_{1} \stackrel{c_{1}}{\longrightarrow} o_{2} \stackrel{c_{2}}{\longrightarrow} \ldots \stackrel{c_{n-1}}{\longrightarrow} o_{n+1} \stackrel{c_{n+1}}{\longrightarrow} o
$$

There is a way of selecting pairs $\left(o_{1}, c_{1}\right), \ldots,\left(o_{n}, c_{n}\right)$ at step 6 of the algorithm (since the length of the corresponding subsequence is equal to $n)$, but since $\left(c_{n+1}, o_{n+1}\right)$ is in $X$ after the step 2 of the initial call, there is a way of selecting that pair at step 6.

Hence, there is a way of selecting pairs $\left(o_{1}, c_{1}\right), \ldots,\left(o_{n+1}, c_{n+1}\right)$ for any given synthesis sequence $S S$.

(2) Let us assume the opposite of the hypothesis, i.e. OSA algorithm terminates with failure, but there is a synthesis sequence $S S$ for object $o$ within node $N$.

From property (1), there is a way of selecting pairs corresponding to the synthesis sequence $S S$ at step 6 in a way that $S O L$ will be equal to $S S$. Besides, the algorithm does not terminate with failure unless all possible pairs are examined (step 9 and step 3 ). If all the possible pairs are examined, then the sequence of pairs coresponding to synthesis sequence $S S$ would be found, and $S S$ would be returned as $S O L$. This is contradictory to our initial assumption, hence the hypothesis is correct.

(3) Let $S O L$ be the sequence returned by the OSA algorithm, and let the size of $S O L$ be $n$. Hence, $S O L$ is of the following form:

$$
o_{1} \stackrel{c_{1}}{\longrightarrow} o_{2} \stackrel{c_{2}}{\longrightarrow} \ldots \stackrel{c_{n-1}}{\longrightarrow} o_{n} \stackrel{c_{n}}{\longrightarrow} o
$$

If $n=1, S O L$ must be returned by step 5 of the OSA algorithm. Since, $c_{1}$ is a conversion capability in $\mathcal{H C}(N)$ (by step 2) and $N \in \operatorname{loc}\left(o_{1}\right)$ (by step 5 ), o can be synthesized from $o_{1}$ using $c_{1}$. Hence, SOL is a sythesis sequence.

Now, let us assume that the hypothesis is true for all solutions with length less than or equal to $n$ returned by the OSA algorithm. Let also $S O L$ be of size $n+1$, i.e $S O L$ is of the following form:

$$
o_{1} \stackrel{c_{1}}{\longrightarrow} o_{2} \stackrel{c_{2}}{\longrightarrow} \ldots \stackrel{c_{n-1}}{\longrightarrow} o_{n} \stackrel{c_{n}}{\longrightarrow} \boldsymbol{o}_{n+1} \stackrel{c_{n+1}}{\longrightarrow} o
$$

The recursive call at step 8 of the OSA algorithm returns a solution sequence $S O L^{\prime}$ for $o_{n+1}$ :

$$
o_{1} \stackrel{c_{1}}{\longrightarrow} o_{2} \stackrel{c_{2}}{\longrightarrow} \ldots \stackrel{c_{n-1}}{\longrightarrow} o_{n} \stackrel{c_{n}}{\longrightarrow} \boldsymbol{o}_{n+1}
$$


This solution sequence is a synthesis sequence, because its length is equal to $n$ (by the inductive hypothesis).

Since, $c_{n+1}$ is a conversion capability in $\mathcal{H C}(N)$ (by step 2), and since $S O L^{\prime}$ is a synthesis sequence for object $o_{n+1}$ (by the inductive hypothesis), $S O L$ is a synthesis sequence for object $o$ within the node $N$.

(4) The set Tried can be of size at most $\operatorname{card}(\mathcal{H C}(N)) \times \operatorname{card}\left(O b j_{N}\right)$. Each time step 7 of the OSA algorithm is executed, the number of elements in Tried increases by 1 - notice that the element being added to Tried cannot alread by in Tried because of the test in Step 5. Therefore, Step 7 of this algorithm can be executed at most $\operatorname{card}(\mathcal{H C}(N)) \times \operatorname{card}\left(O b j_{N}\right)$.

Proof of Theorem 3.2. (1) The solution returned by the OptOSA algorithm is a valid synthesis sequence. Let solution returned by OptOSA be of the following form:

$$
o_{1} \stackrel{c_{1}}{\longrightarrow} o_{2} \stackrel{c_{2}}{\longrightarrow} \ldots \stackrel{c_{n-1}}{\longrightarrow} \boldsymbol{o}_{n} \stackrel{c_{n}}{\longrightarrow} \boldsymbol{o}
$$

The initial object $o_{1}$ and all the conversion operators $c_{1}, \ldots, c_{n}$ are available in node $N$ (by steps 3 and 6(a)iv). Hence, the solution is a valid synthesis sequence.

Now, let the synthesis sequence returned by the OptOSA algorithm be $S S$, and let $S S$ be a sub-optimal sequence. Hence, there exists a synthesis sequence $S S^{\prime}$ with a lower cost.

Let $S S$ be of the following form:

$$
o_{1} \stackrel{c_{1}}{\longrightarrow} o_{2} \stackrel{c_{2}}{\longrightarrow} \ldots \stackrel{c_{n-1}}{\longrightarrow} o_{n} \stackrel{c_{n}}{\longrightarrow} o
$$

And let $S S^{\prime}$ be of the following form:

$$
o_{1}^{\prime} \stackrel{c_{1}^{\prime}}{\longrightarrow} o_{2}^{\prime} \stackrel{c_{2}^{\prime}}{\longrightarrow} \ldots \stackrel{c_{m-1}^{\prime}}{\longrightarrow} o_{m}^{\prime} \stackrel{c_{m}^{\prime}}{\longrightarrow} o
$$

Since $S S$ is suboptimal, but $S S^{\prime}$ is optimal, $\operatorname{cost}(S S)>\operatorname{cost}\left(S S^{\prime}\right)$. Furthermore, since the cost of each step in the synthesis is non-negative, the following observations hold:

$$
\begin{gathered}
\operatorname{cost}(S S)>\operatorname{cost}\left(o_{1}^{\prime} \stackrel{c_{1}^{\prime}}{\longrightarrow} \ldots \stackrel{c_{m-1}^{\prime}}{\longrightarrow} o_{m}^{\prime}\right) \\
\operatorname{cost}(S S)>\operatorname{cost}\left(o_{1}^{\prime} \stackrel{c_{1}^{\prime}}{\longrightarrow} \ldots \stackrel{c_{m-2}^{\prime}}{\longrightarrow} o_{m-1}^{\prime}\right) \\
\ldots \ldots \\
\operatorname{cost}(S S)>\operatorname{cost}\left(o_{1}^{\prime} \stackrel{c_{1}^{\prime}}{\longrightarrow} o_{2}^{\prime}\right)
\end{gathered}
$$

Since $\left(o_{1}^{\prime}, c_{1}^{\prime}\right)$ is in $T^{0}(o)$ and it has a lower cost, $\left(o_{1}^{\prime}, c_{1}^{\prime}\right)$ is going to be examined before $\left(o_{n}, c_{n}\right)$. Then, $\left(o_{2}^{\prime}, c_{2}^{\prime}\right)$ will be in $T^{1}(o)$ and it will have a lower cost than $\left(o_{n}, c_{n}\right)$, hence it will be evaluated before $\left(o_{n}, c_{n}\right)$. Similarly, $\left(o_{m}^{\prime}, c_{m}^{\prime}\right)$ will be in $T^{m-1}(o)$ and it will have a lower cost than $\left(o_{n}, c_{n}\right)$. So, $\left(o_{m}^{\prime}, c_{m}^{\prime}\right)$ will be evaluated before $\left(o_{n}, c_{n}\right)$ and $S S^{\prime}$ will be returned instead of $S S$. This is a contradiction of the initial assumption, hence if OptOSA returns a synthesis sequence, then this sequence is optimal. 
(2) If OptOSA returns failure, it must do so at step 5. Hence, in the case of failure, the linked list $X$ must be empty. $X$ becomes empty when every possible sequence is checked and no solution is found. Hence, if OptOSA returns failure, then there is no solution.

If there is no synthesis sequence for the requested object, then OptOSA cannot return any solution except failure, because by the previous property, if OptOSA returns a solution, then there exists a synthesis sequence for the requested object.

¿From the above two paragraphs, we can conclude that, the OptOSA algorithm returns with failure iff there is no synthesis sequence for object $o$ in node $N$.

(3) Property 1 above already shows that if OptOSA returns a solution, then the solution is an optimal one. Hence, showing that if there is a synthesis sequence then OptOSA returns a non-empty solution sequence is enough to prove our claim.

Let us assume that there is a synthesis sequence $S S$ for the object $o$ within the node $N$, and let $S S$ be of the following form:

$$
o_{1} \stackrel{c_{1}}{\longrightarrow} o_{2} \stackrel{c_{2}}{\longrightarrow} \ldots \stackrel{c_{n-1}}{\longrightarrow} o_{n} \stackrel{c_{n}}{\longrightarrow} o
$$

Since $o_{1}$ is in node $N$, there is a quadruple $q$ of the form $\left(o_{1}, \operatorname{size}\left(o_{1}\right), 0, o_{1}\right)$ in $X$ after step 4 .

Let us assume that there are $k$ quadruples in $X$ before $q: q_{1}, q_{2}, \ldots, q_{k}$. For the sake of the argument, let us also assume that none of these quadruples leads to a synthesis sequence.

$q_{1}$ will be the first quadruple selected in step 6 of the algorithm. $q_{1}$ will result in at most $C$ new quadruples where $C$ is the number of conversion functions available in node $N$. In the worst case, $C$ new quadruples will get in front of $q$ in the list, and one quadruple $\left(q_{1}\right)$, will be deleted. Hence the total number of quadruples before $q$ in $X$ is now $\leq k+C$. This is obviously larger than the initial value $k$. However, this fact cannot result in an infinite computation, because of the following:

- The sizes of the objects are integers.

- The if condition in 6(a)iv prevents objects to be recreated with larger (or same) sizes during the synthesis.

- There is a finite number of distinct types.

The first two properties guarantee that an object o.t can be recreated at most size(o.t) times during the synthesis sequence.

Let $\rho(t, i)$ be equal to $\max (\{$ size_ratio $(c) \mid c \in \mathcal{H C}(N)$ and $c(o . t)=o . i\})$ (if the set $\{$ size_ratio $(c) \mid c \in$ $\mathcal{H C}(N)$ and $c(o . t)=o . i\}$ is empty, then let $\rho(t, i)$ be 0$)$. Let also the number of distinct types be $T$ (by the third property $T$ is finite). Then, an object o.t can lead to less than

$$
\sum_{i=1}^{T} \rho(t, i) \times \operatorname{size}(o . t)
$$

new objects during the object synthesis.

Hence, a quadruple can cause only a finite amount of quadruples be created and placed in $X$. Hence, quadruples $q_{1}$ though $q_{k}$ will cause only a finite new of new quadruples to be placed before $q$. Hence, eventually, all those quadruples will be evaluated and consumed and $q$ will be processed, and as a 
result of this $q^{\prime}=\left(o_{2}\right.$, Size,Cost, $\left.o_{1} \stackrel{c_{1}}{\longrightarrow} o_{2}\right)$ will be placed in $X$ (Size is the size of $o_{2}$, and Cost is the cost of synthesizing $\left.o_{2}\right)$.

Using the above argument iteratively, it is possible to show that the synthsis sequence $S S$ will eventually be found and returned by the OptOSA algorithm.

Proof of Theorem 3.3. Follows along the same lines as the proof of Theorem 3.1. 